Article

\title{
Synthesis and Antifungal Activity of Novel 3-Caren-5-One Oxime Esters
}

\author{
Min Huang, Wen-Gui Duan *, Gui-Shan Lin *, Kun Li and Qiong Hu \\ School of Chemistry and Chemical Engineering, Guangxi University, Nanning 530004, Guangxi, China; \\ gaominhuang@mail.gxu.cn (M.H.); k1756004166@sina.com (K.L.); hq460175625@163.com (Q.H.) \\ * Correspondence: wgduan@gxu.edu.cn (W.-G.D.); gxlinguas@sina.com (G.-S.L.); \\ Tel.: +86-771-323-9910 (W.-G.D.); Fax: +86-771-323-3718 (W.-G.D.)
}

Received: 28 August 2017; Accepted: 8 September 2017; Published: 12 September 2017

\begin{abstract}
A series of novel 3-caren-5-one oxime esters were designed and synthesized by multi-step reactions in an attempt to develop potent antifungal agents. Two $E-Z$ stereoisomers of the intermediate 3-caren-5-one oxime were separated by column chromatography for the first time. The structures of all the intermediates and target compounds were confirmed by UV-Vis, FTIR, NMR, ESI-MS, and elemental analysis. The antifungal activity of the target compounds was preliminarily evaluated by the in vitro method against Fusarium oxysporum f. sp. cucumerinum, Physalospora piricola, Alternaria solani, Cercospora arachidicola, Gibberella zeae, Rhizoeotnia solani, Bipolaris maydis, and Colleterichum orbicalare at $50 \mu \mathrm{g} / \mathrm{mL}$. The target compounds exhibited best antifungal activity against P. piricola, in which compounds $(Z)-\mathbf{4 r}(\mathrm{R}=\beta$-pyridyl $),(Z)-\mathbf{4 q}(\mathrm{R}=\alpha$-thienyl $),(E)-\mathbf{4 \mathbf { f } ^ { \prime }}(\mathrm{R}=p$-F $\mathrm{Ph})$, (Z)-4i $(\mathrm{R}=m-\mathrm{Me} \mathrm{Ph}),(Z)-4 \mathbf{j}(\mathrm{R}=p$-Me Ph), and $(Z)-4 \mathbf{p}(\mathrm{R}=\alpha$-furyl) had inhibition rates of $97.1 \%$, $87.4 \%, 87.4 \%, 85.0 \%, 81.9 \%$, and $77.7 \%$, respectively, showing better antifungal activity than that of the commercial fungicide chlorothanil. Also, compound $(\mathrm{Z})-\mathbf{4 r}(\mathrm{R}=\beta$-pyridyl) displayed remarkable antifungal activity against all the tested fungi, with inhibition rates of $76.7 \%, 82.7 \%, 97.1 \%, 66.3 \%$, $74.7 \%, 93.9 \%, 76.7 \%$ and $93.3 \%$, respectively, showing better or comparable antifungal activity than that of the commercial fungicide chlorothanil. Besides, the $E-Z$ isomers of the target oxime esters were found to show obvious differences in antifungal activity. These results provide an encouraging framework that could lead to the development of potent novel antifungal agents.
\end{abstract}

Keywords: 3-carene; 3-caren-5-one; 3-caren-5-one oxime ester; $E$-Z stereoisomer; antifungal activity

\section{Introduction}

3-Carene, a naturally occurring bicyclic monoterpene containing a carbon-carbon double bond and a gem-dimethylcyclopropane ring, is a constituent of many essential oils and turpentine oils $[1,2]$. It was reported that the isomeric mixture of 3-carene showed a broad spectrum of activities, such as antimicrobial [3-6], anticancer [6], semiochemical [7,8], antioxidant [6,9], and fumigant activities [10]. Because of its special structure and bioactive activities, screening of structurally modified 3-carene derivatives for their bioactivity has received considerable attention. For instance, stable, potent, and selective sphingosine-1-phosphate receptor $1\left(\mathrm{~S}_{1} \mathrm{P}_{1}\right)$ agonists were successively synthesized by using (+)-3-carene as starting material [11]. Ingenol and (+)-phorbol, two crucial natural products with various biological activities, especially anticancer, have been also synthesized from inexpensive (+)-3-carene [12-15]. On the other hand, oxime esters as an important class of bioactive compounds for agrochemical and pharmaceutical use were investigated extensively by chemists. It was reported that oxime esters exhibited insecticidal [16,17], herbicidal [18], antiviral [19], antitumor [20-22], and antibacterial activities [23-25]. For example, a series of 3-ethoxy-4-hydroxybenzaldehyde oxime esters were synthesized and evaluated for their in vitro antifungal activity against three pathogenic fungi and antibacterial activity against three bacterial strains, and the structure-activity 
relationship was also preliminarily summarized [26]. In continuation of our interest in the bioactive properties of natural product-based compounds [27-33], a series of novel 3-caren-5-one oxime esters were designed and synthesized by integrating bioactive oxime esters into the skeleton of 3-carene. Structural characterization and antifungal evaluation of all the title compounds were carried out as well. In addition, $\mathrm{Cu}(\mathrm{II}) \mathrm{HY}$ zeolite was first employed as catalyst in the oxidative preparation of 3-caren-5-one, and the E-Z stereo-isomers of 3-caren-5-one oxime were also separated for the first time by column chromatography.

\section{Results and Discussion}

\subsection{Synthesis and Characterization}

As illustrated in the Scheme 1, 3-caren-5-one (2) was prepared by oxidation of 3-carene (1, isomeric mixture) using TBHP (tert-butyl hydroperoxide) as oxidant in a 30\% yield (GC). In this process, $\mathrm{Cu}(\mathrm{II}) \mathrm{HY}$, in which $\mathrm{Cu}(\mathrm{II})$ ions were incorporated into $\mathrm{HY}$ zeolite, was chosen as a heterogeneous catalyst to increase the selectivity for $\mathbf{2}$ due to the shape-selectivity of this mesoporous material [34] and the activation capacity of copper ion to saturated $\mathrm{C}-\mathrm{H}$ [35-37]. After the reaction was completed, this catalyst could easily be separated from the reaction system by filtration, and could be recycled several times.
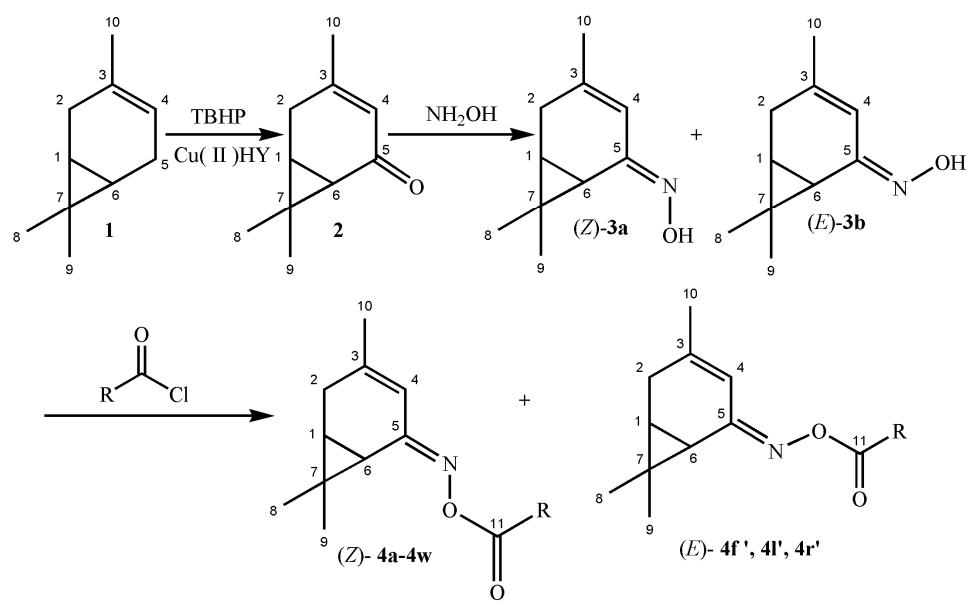

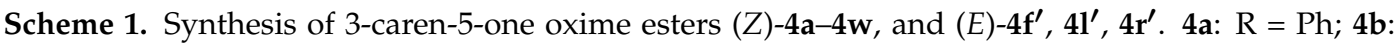
$\mathrm{R}=o-\mathrm{Cl} \mathrm{Ph} ; 4 \mathrm{c}: \mathrm{R}=m-\mathrm{Cl} \mathrm{Ph} ; 4 \mathrm{~d}: \mathrm{R}=p-\mathrm{Cl} \mathrm{Ph} ; 4 \mathrm{e}: \mathrm{R}=o-\mathrm{F} \mathrm{Ph} 4 \mathrm{f}: \mathrm{R}=p-\mathrm{F} \mathrm{Ph} ; 4 \mathrm{~g}: \mathrm{R}=o-\mathrm{OMe} \mathrm{Ph} ; 4 \mathbf{h}:$ $\mathrm{R}=o-\mathrm{Me} \mathrm{Ph} ; 4 \mathbf{i}: \mathrm{R}=m-\mathrm{Me} \mathrm{Ph} ; \mathbf{j}: \mathrm{R}=p-\mathrm{Me} \mathrm{Ph} ; \mathbf{k}: \mathrm{R}=2,4-\mathrm{Cl} \mathrm{Ph} ; 4 \mathbf{l}: \mathrm{R}=2,3-\mathrm{Cl} \mathrm{Ph} ; 4 \mathrm{~m}: \mathrm{R}=p-\mathrm{CH}_{2} \mathrm{Cl}$ $\mathrm{Ph} ; 4 \mathrm{n}: \mathrm{R}=$ cyclopentyl; 4o: $\mathrm{R}=$ cyclohexyl; 4p: $\mathrm{R}=\alpha$-furyl; 4q: $\mathrm{R}=\alpha$-thienyl; 4r: $\mathrm{R}=\beta$-pyridyl; 4s: $\mathrm{R}=\alpha$-Cl- $\beta$-pyridyl; 4t: $\mathrm{R}=\mathrm{n}$-pentyl; $4 \mathbf{u}: \mathrm{R}=$ ethyl; $4 \mathbf{v}: \mathrm{R}=\mathrm{n}$-propyl; $4 \mathbf{w}: \mathrm{R}=\mathrm{n}$-butyl; $4 \mathrm{f}^{\prime}: \mathrm{R}=p$-F Ph; $41^{\prime}: \mathrm{R}=2,3-\mathrm{Cl} ; 4 \mathrm{r}^{\prime}: \mathrm{R}=\beta$-pyridyl.

3-Caren-5-one oxime (3, isomeric mixture) was prepared by oximation of compound 2 with $\mathrm{NH}_{2} \mathrm{OH} \cdot \mathrm{HCl}$ in a mixed solvent $\left(\mathrm{EtOH}: \mathrm{H}_{2} \mathrm{O}=5: 1, v / v\right)$. Sodium acetate was added into the reaction system as an additive to neutralize $\mathrm{HCl}$ and form a buffer system. Two stereoisomers, (Z)-3-caren-5-one oxime ((Z)-3a) and $(E)-3$-caren-5-one oxime $((E)-\mathbf{3} \mathbf{b})$, were isolated from compound $\mathbf{3}$ (3a:3b $=7: 1, w / w$, $\mathrm{GC})$. In the ${ }^{1} \mathrm{H}-\mathrm{NMR}$ spectrum of $(\mathrm{Z})-3 \mathrm{a}$, the olefinic proton $\left(\mathrm{C}_{4}-\mathrm{H}\right)$ in the 3-carene skeleton showed signals at about 5.88 and the signals of the $\mathrm{C}_{6}-\mathrm{H}$ appeared at $1.99 \mathrm{ppm}$. However, the corresponding signals of another isomer (E)-3b were shifted to $6.65 \mathrm{ppm}$ and $1.50 \mathrm{ppm}$, respectively (Figure 1). Due to the hydrogen bond interaction of the hydroxyl group oxygen with the olefinic proton $\left(\mathrm{C}_{4}-\mathrm{H}\right)$ or the $\mathrm{C}_{6}-\mathrm{H}$, the signal of olefinic proton $\left(\mathrm{C}_{4}-\mathrm{H}\right)$ in the $(E)$-isomer and the signal of the $\mathrm{C}_{6}-\mathrm{H}$ in the $(\mathrm{Z})$-isomer shifted to downfield [38]. The structures of the two stereo-isomers were further confirmed by NOESY spectra. In the NOESY spectrum of (Z)-3a, no peak related to the coupling of hydroxyl hydrogen with $\mathrm{C}_{4}-\mathrm{H}$ was observed, while a peak corresponding to this space interaction in $(E)-3 \mathbf{b}$ was found (Figure 2). 


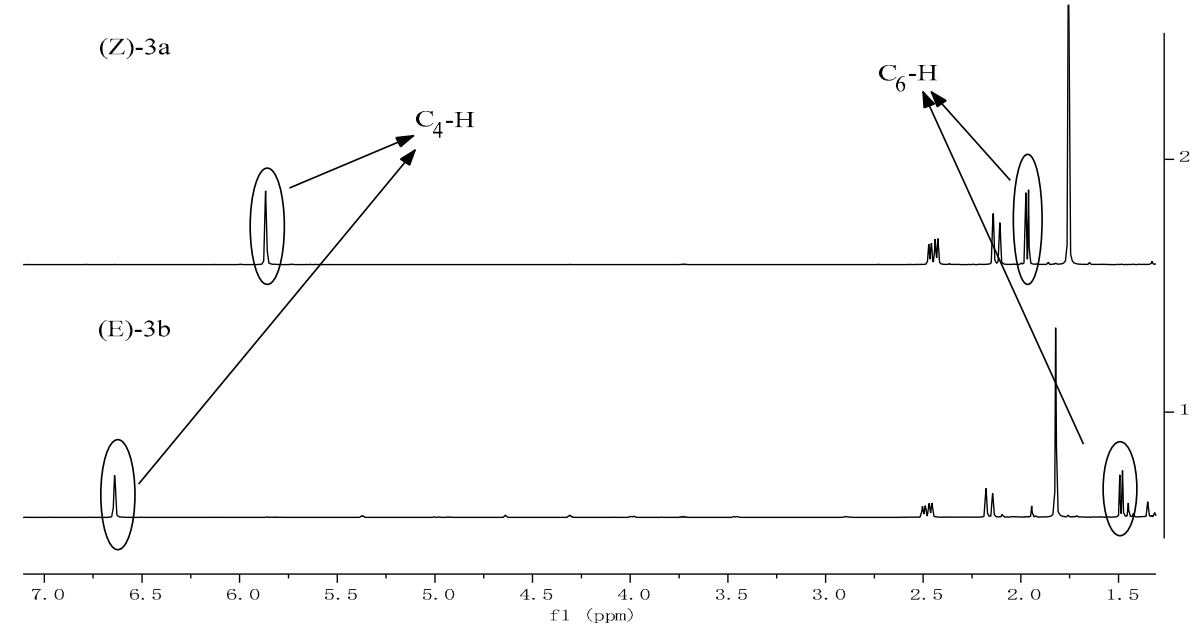

Figure 1. ${ }^{1} \mathrm{H}-\mathrm{NMR}$ spectra of $(Z)-3 \mathbf{a}$ and $(E)-\mathbf{3 b}$.
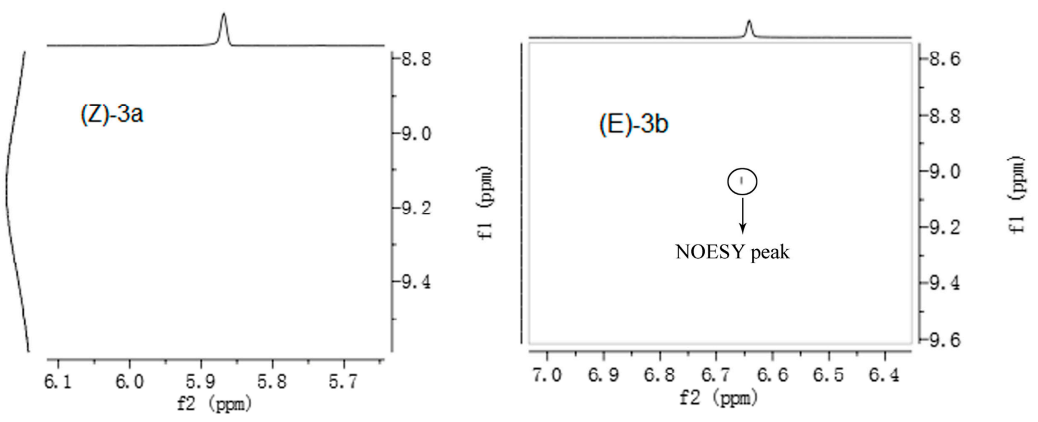

Figure 2. The coupling of $\mathrm{C}_{4}-\mathrm{H}$ and hydroxyl hydrogen in ${ }^{1} \mathrm{H}^{-1} \mathrm{H}$ NOESY spectra.

The target oxime esters were characterized by UV-Vis, IR, ${ }^{1} \mathrm{H}-\mathrm{NMR},{ }^{13} \mathrm{C}-\mathrm{NMR}$, ESI-MS, and elemental analysis, and all the related spectra can be found in the Supplementary Materials. It was found that the molar extinction coefficient of all target compounds ranged from 3.89 to $4.42(\log \varepsilon)$, implying that a conjugated system existed in these compounds. In the IR spectra, the weak absorption bands at about $3018 \mathrm{~cm}^{-1}$ were attributed to the stretching vibrations of the unsaturated $\mathrm{C}-\mathrm{H}$ in the 3 -carene moiety. The strong absorption bands at about $1738 \mathrm{~cm}^{-1}$ were assigned to the vibrations of $\mathrm{C}=\mathrm{O}$ in the ester moiety. The moderate absorption peaks at about $1649 \mathrm{~cm}^{-1}$ were due to the vibrations of $\mathrm{C}=\mathrm{N}$. In the ${ }^{1} \mathrm{H}-\mathrm{NMR}$ spectra, the protons of benzene ring showed signals at 7.10-8.20 ppm. The olefinic protons of the 3-carene scaffold showed signals at about $6.10 \mathrm{ppm}$ for the (Z)-isomers and the (E)-isomers showed signals at about $6.60 \mathrm{ppm}$. The protons bonded to the saturated carbons displayed signals in the range of $0.50-3.00 \mathrm{ppm}$. The ${ }^{13} \mathrm{C}-\mathrm{NMR}$ spectra of the target compounds showed peaks for the olefinic carbons of the 3-carene moiety at 117.92-119.05 ppm $\left(C_{4}\right)$ and $147.49-149.74 \mathrm{ppm}\left(\mathrm{C}_{3}\right)$ for the $(Z)$-isomers, however, the corresponding signals for $(E)$-isomers showed at about $113.20\left(C_{4}\right)$ ppm and $153.03 \mathrm{ppm}\left(\mathrm{C}_{3}\right)$. The other saturated carbons displayed signals in the region of 13.59-55.94 $\mathrm{ppm}$. The other signal peaks were correlated to the carbons of $\mathrm{C}=\mathrm{O}, \mathrm{C}=\mathrm{N}$, and the benzene ring. The molecular weights of the intermediates and target oxime esters were confirmed by the ESI-MS.

\subsection{Antifungal Activity}

The antifungal activities of the target compounds $(Z)-\mathbf{4 a}-\mathbf{4} \mathbf{w}$ and $(E)-\mathbf{4 \mathbf { f } ^ { \prime }}$ were evaluated by in vitro method against fusarium wilt on cucumber (Fusarium oxysporum $\mathrm{f}$. sp. cucumerinum), speckle on peanut (Cercospora arachidicola), apple ring rot (Physalospora piricola), tomato early blight (Alternaria solani), wheat scab (Gibberella zeae), rice sheath blight (Rhizoeotnia solani), corn southern leaf blight (Bipolaris maydis), and watermelon anthracnose (Colleterichum orbicalare) at $50 \mu \mathrm{g} / \mathrm{mL}$. The results are listed in Table 1. 
Table 1. Antifungal activity of the target compounds (Z)-4a-4w and (E)-4f $\mathbf{f}^{\prime}$ at $50 \mu \mathrm{g} / \mathrm{mL}$.

\begin{tabular}{|c|c|c|c|c|c|c|c|c|}
\hline \multirow{2}{*}{ Compounds } & \multicolumn{8}{|c|}{ Relative Inhibition Rate (\%) against the Tested Fungi } \\
\hline & F. oxysporum f. sp. cucumerinum & C. arachidicola & P. piricola & A. solani & G. zeae & R. solani & B. myadis & C. orbicalare \\
\hline$(Z)-4 a(R=P h)$ & 28.8 & 35.0 & 69.4 & 13.7 & 21.8 & 0 & 32.2 & 43.3 \\
\hline$(\mathrm{Z})-\mathbf{4} \mathbf{b}(\mathrm{R}=o-\mathrm{ClPh})$ & 16.3 & 30.0 & 0 & 0 & 21.8 & 18.3 & 23.9 & 30.5 \\
\hline$(\mathrm{Z})-4 \mathrm{c}(\mathrm{R}=m-\mathrm{ClPh})$ & 22.5 & 0 & 44.4 & 0 & 21.8 & 17.1 & 26.7 & 33.1 \\
\hline (Z)-4d $(\mathrm{R}=p-\mathrm{ClPh})$ & 28.8 & 0 & 28.8 & 0 & 19.8 & 0 & 29.4 & 33.1 \\
\hline$(\mathrm{Z})-4 \mathrm{e}(\mathrm{R}=o-\mathrm{FPh})$ & 31.9 & 45.0 & 41.3 & 35.9 & 37.5 & 35.0 & 26.7 & 45.9 \\
\hline (Z)-4f (R = p-FPh) & 25.6 & 15.0 & 30.7 & 0.0 & 15.9 & 0 & 26.7 & 23.8 \\
\hline (Z)-4g $(\mathrm{R}=o-\mathrm{OMePh})$ & 25.6 & 0 & 60.0 & 21.1 & 13.9 & 0 & 26.7 & 35.6 \\
\hline$(\mathrm{Z})-4 \mathrm{~h}(\mathrm{R}=o-\mathrm{MePh})$ & 31.9 & 50.0 & 28.8 & 21.1 & 29.6 & 38.6 & 29.4 & 43.3 \\
\hline$(\mathrm{Z})-4 \mathbf{i}(\mathrm{R}=m-\mathrm{MePh})$ & 19.4 & 15.0 & 85.0 & 0 & 12.0 & 0 & 23.9 & 0 \\
\hline$(\mathrm{Z})-4 \mathbf{j}(\mathrm{R}=p-\mathrm{MePh})$ & 38.1 & 20.0 & 81.9 & 24.8 & 17.8 & 0 & 35.0 & 53.6 \\
\hline$(\mathrm{Z})-4 \mathbf{k}(\mathrm{R}=2,4-\mathrm{ClPh})$ & 28.8 & 55.0 & 16.3 & 13.7 & 17.8 & 18.3 & 23.9 & 53.6 \\
\hline$(\mathrm{Z})-41(\mathrm{R}=2,3-\mathrm{ClPh})$ & 25.6 & 15.0 & 28.8 & 17.4 & 19.8 & 0 & 21.1 & 43.3 \\
\hline (Z)-4m (R = p-CH $\left.\mathrm{CH}_{2} \mathrm{ClPh}\right)$ & 56.9 & 20.0 & 41.3 & 0 & 19.8 & 0 & 21.1 & 40.8 \\
\hline (Z)-4n ( $\mathrm{R}=$ cyclo-pentyl $)$ & 22.5 & 35.0 & 0 & 13.7 & 19.8 & 0 & 23.9 & 51.0 \\
\hline (Z)-4o $(\mathrm{R}=$ cyclo-hexyl $)$ & 25.6 & 30.0 & 28.8 & 21.1 & 15.9 & 16.0 & 23.9 & 40.8 \\
\hline$(\mathrm{Z})-4 \mathbf{p}(\mathrm{R}=\alpha$-furyl $)$ & 60.0 & 64.5 & 77.7 & 53.8 & 36.5 & 54.6 & 43.3 & 54.4 \\
\hline (Z)-4q (R = $\alpha$-thienyl) & 48.9 & 37.3 & 87.4 & 41.3 & 33.5 & 42.1 & 33.8 & 48.9 \\
\hline (Z)-4r $(\mathrm{R}=\beta$-pyridyl $)$ & 76.7 & 82.7 & 97.1 & 66.3 & 74.7 & 93.9 & 76.7 & 93.3 \\
\hline (Z)-4s $(\mathrm{R}=\alpha$-Cl- $\beta$-pyridyl $)$ & 15.6 & 37.3 & 55.2 & 41.3 & 54.1 & 63.6 & 24.3 & 21.1 \\
\hline (Z)-4t $(\mathrm{R}=n$-pentyl) & 21.1 & 19.1 & 58.4 & 35.0 & 48.2 & 67.1 & 24.3 & 21.1 \\
\hline (Z)-4u (R = n-ethyl) & 15.6 & 19.1 & 58.4 & 28.8 & 48.2 & 36.8 & 29.0 & 21.1 \\
\hline (Z)-4v (R = n-propyl) & 15.6 & 28.2 & 32.6 & 41.3 & 57.1 & 54.6 & 24.3 & 21.1 \\
\hline (Z)-4w ( $\mathrm{R}=n$-butyl) & 15.6 & 19.1 & 51.9 & 22.5 & 18.8 & 18.9 & 19.5 & 21.1 \\
\hline$(E)-4 \mathbf{f}^{\prime}(\mathrm{R}=p-\mathrm{FPh})$ & 60.0 & 64.5 & 87.4 & 53.8 & 65.9 & 76.1 & 76.7 & 54.4 \\
\hline$(Z)-3 a$ & 15.6 & 23.6 & 51.9 & 35.0 & 18.8 & 24.3 & 24.3 & 26.7 \\
\hline$(E)-\mathbf{3 b}$ & 15.6 & 23.6 & 51.9 & 22.5 & 18.8 & 27.9 & 19.5 & 21.1 \\
\hline 2 & 21.1 & 19.1 & 45.5 & 16.3 & 24.7 & 18.9 & 24.3 & 15.6 \\
\hline Chlorothanil & 100 & 73.3 & 75.0 & 73.9 & 73.1 & 96.1 & 90.4 & 91.3 \\
\hline
\end{tabular}


It was found that, at $50 \mu \mathrm{g} / \mathrm{mL}$, all the target compounds presented obviously different antifungal activity against the eight tested fungi. Compared with the intermediates $\mathbf{2}, \mathbf{3 a}$ and $\mathbf{3 b}$, some of the target compounds showed enhanced activities after esterification. On the whole, most of the target compounds exhibited best antifungal activity against $P$. piricola, in which compounds $(Z)-4 \mathbf{r}$ $(\mathrm{R}=\beta$-pyridyl), (Z)-4q ( $\mathrm{R}=\alpha$-thienyl), (E)-4f' $(\mathrm{R}=p$-F Ph), (Z)-4i $(\mathrm{R}=m-\mathrm{Me} \mathrm{Ph}),(Z)-\mathbf{4 j}(\mathrm{R}=p$-Me $\mathrm{Ph}$ ), and $(Z)-4 p(\mathrm{R}=\alpha$-furyl) had inhibition rates of $97.1 \%, 87.4 \%, 87.4 \%, 85.0 \%, 81.9 \%$, and $77.7 \%$, respectively, showing better antifungal activity than that of the commercial fungicide chlorothanil with the inhibition rate of $75.0 \%$. It was also found that compound $(Z)-4 \mathbf{r}(\mathrm{R}=\beta$-pyridyl) displayed remarkable antifungal activity against all the tested fungi, with inhibition rates of $76.7 \%, 82.7 \%, 97.1 \%$, $66.3 \%, 74.7 \%, 93.9 \%, 76.7 \%$ and $93.3 \%$, respectively, showing better or comparable antifungal activity than that of the commercial fungicide chlorothanil. Therefore, compound $(Z)-4 r(R=\beta$-pyridyl) is a lead compound worthy of further investigation. Furthermore, it was found that $(E)$-isomer $4 \mathbf{f}^{\prime}(\mathrm{R}=p$-F $\mathrm{Ph}$ ) exhibited much better antifungal activity than the corresponding (Z)-isomer $4 \mathrm{f}(\mathrm{R}=p$-F $\mathrm{Ph})$ against all the tested fungi. For instance, $(E)$-isomer $\mathbf{4 f ^ { \prime }}(64.5 \%)$ showed 4.3 times that of $\mathbf{4 f}(Z)$-isomer $(15.0 \%)$ against $C$. arachidicola, and the two isomers displayed $76.1 \%$ and $0.0 \%$ inhibition rates, respectively, against $R$. solani. The obvious difference in antifungal activity between $E-Z$ isomers is very meaningful and requires further studies in photoisomerization and drug resistance.

\section{Experimental Section}

\subsection{General Information}

The GC analysis was performed on an Agilent 6890 GC (Agilent Technologies Inc., Santa Clara, CA, USA) equipped with a HP-1 $(30 \mathrm{~m}, 0.530 \mathrm{~mm}, 0.88 \mu \mathrm{m})$ column. IR spectra were recorded $(\mathrm{KBr}$ pellet method) on a Nicolet iS50 FT-IR spectrometer (Thermo Scientific Co., Ltd., Madison, WI, USA). NMR spectra (including ${ }^{1} \mathrm{H}-\mathrm{NMR},{ }^{13} \mathrm{C}-\mathrm{NMR}, \mathrm{NOESY}$ ) were recorded in $\mathrm{CDCl}_{3}$ solvent on a Bruker Avance III HD 600 MHz spectrometer (Bruker Co., Ltd., Zurich, Switzerland) and chemical shifts are expressed in ppm $(\delta)$ downfield relative to TMS as an internal standard. MS spectra were obtained by means of the electrospray ionization (ESI) method on TSQ Quantum Access MAX HPLC-MS instrument (Thermo Scientific Co., Ltd., Waltham, MA, USA). Elemental analyses were measured using a PE 2400 II elemental analyzer (Perkin-Elmer Instruments Co., Ltd., USA). The UV spectra were measured on a Shimadzu UV-1800 spectrophotometer (Shimadzu Corp., Kyoto, Japan). Melting points were determined on a MP420 automatic melting point apparatus (Hanon Instruments Co., Ltd., Jinan, China) and were not corrected. Ultrasonic irradiation was carried out on XO-SM50 ultrasonic microwave reaction system (Nanjing Xianou Instrument Manufacturing Co., Ltd., Nanjing, China). 3-Carene (GC purity 98\%, isomeric mixture) was provided by Wuzhou Pine Chemicals Co., Ltd.

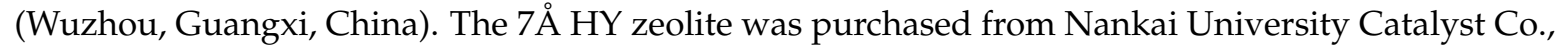
Ltd. (Tianjin, China). Other reagents were purchased from commercial suppliers and used as received.

\subsection{Preparation of Catalyst}

$\mathrm{Cu}(\mathrm{II}) \mathrm{HY}$ zeolite catalyst used for the synthesis of 3-caren-5-one was prepared by the incipient wetness method [39]. HY zeolite with pore diameter of $7 \AA$ was dried at $120{ }^{\circ} \mathrm{C} 3 \mathrm{~h}$ under air atmosphere. Then, a solution of $\mathrm{Cu}\left(\mathrm{NO}_{3}\right)_{2} \cdot 3 \mathrm{H}_{2} \mathrm{O}(5.7 \mathrm{~g})$ in deionized water based on the saturated water absorption amount of the zeolite $(10.0 \mathrm{~g})$ was added dropwise to the dried zeolite, with constant stirring and grinding. The resulting mixture was aged for $24 \mathrm{~h}$, and then dried in vacuum at $120{ }^{\circ} \mathrm{C}$ for $2 \mathrm{~h}$. The powder sample was calcinated at $550{ }^{\circ} \mathrm{C}$ for $4 \mathrm{~h}$ under air atmosphere.

\subsection{Synthesis of 3-Caren-5-One (2)}

3-Carene (10 mL, $63 \mathrm{mmol}), \mathrm{Cu}(\mathrm{II}) \mathrm{HY}$ zeolite catalyst $(2.7 \mathrm{~g})$ and $\mathrm{CH}_{3} \mathrm{CN}(20 \mathrm{~mL})$ were mixed and then irradiated under ultrasonic wave for $40 \mathrm{~min}$. When the mixture was heated to $45^{\circ} \mathrm{C}$, a mixed solution of $40 \mathrm{~mL}$ tert-butyl hydroperoxide (70\% in water, wt \%) $(288 \mathrm{mmol})$ and $20 \mathrm{~mL} \mathrm{CH}_{3} \mathrm{CN}_{\text {was }}$ 
added to the reaction system by the way of constant flowing at a rate of $1 \mathrm{~mL} / \mathrm{min}$. Afterwards, the reaction mixture was stirred for $18 \mathrm{~h}$ at $45^{\circ} \mathrm{C}$. When the reaction was completed, the reaction mixture was cooled to room temperature and appropriate amount of $\mathrm{Na}_{2} \mathrm{~S}_{2} \mathrm{O}_{3}$ was added into it to destroy the unreacted tert-butyl hydroperoxide. The solvent $\mathrm{CH}_{3} \mathrm{CN}$ was removed by vacuum distillation, and the residue was then extracted three times with EtOAc. The combined organic phase was washed with saturated sodium chloride and deionized water, respectively, and dried over with anhydrous $\mathrm{Na}_{2} \mathrm{SO}_{4}$. After the EtOAc was removed in vacuum, the crude product was purified by silica gel column chromatography (EtOAc-petroleum ether $=1: 30, v / v)$ to obtain pale yellow liquid compound 2. Yield 20\%. UV-Vis (EtOH) $\lambda_{\max }(\log \varepsilon): 226.50(4.4 .16) \mathrm{nm}$; IR (thin film, $\left.\mathrm{cm}^{-1}\right): 3029$ (w, =C-H), 1655 $(\mathrm{s}, \mathrm{C}=\mathrm{O}) ;{ }^{1} \mathrm{H}-\mathrm{NMR} \delta=5.83\left(\mathrm{~s}, 1 \mathrm{H}, \mathrm{C}_{4}-\mathrm{H}\right), 2.64\left(\mathrm{dd}, J=20.4,8.5 \mathrm{~Hz}, 1 \mathrm{H}, \mathrm{C}_{2}-\mathrm{H}_{\mathrm{a}}\right), 2.33(\mathrm{~d}, J=20.8 \mathrm{~Hz}, 1 \mathrm{H}$, $\left.\mathrm{C}_{2}-\mathrm{H}_{\mathrm{b}}\right), 1.87\left(\mathrm{~s}, 3 \mathrm{H}, \mathrm{C}_{3}-\mathrm{CH}_{3}\right), 1.56\left(\mathrm{~d}, J=7.8 \mathrm{~Hz}, 1 \mathrm{H}, \mathrm{C}_{6}-\mathrm{H}\right), 1.45\left(\mathrm{t}, J=8.0 \mathrm{~Hz}, 1 \mathrm{H}, \mathrm{C}_{1}-\mathrm{H}\right), 1.19(\mathrm{~s}, 3 \mathrm{H}$, $\left.\mathrm{C}_{7}-\mathrm{CH}_{3}\right), 1.04\left(\mathrm{~s}, 3 \mathrm{H}, \mathrm{C}_{7}-\mathrm{CH}_{3}\right) ;{ }^{13} \mathrm{C}-\mathrm{NMR} \delta=196.67\left(\mathrm{C}_{5}\right), 158.96\left(\mathrm{C}_{3}\right), 126.40\left(\mathrm{C}_{4}\right), 32.85\left(\mathrm{C}_{6}\right), 28.43\left(\mathrm{C}_{9}\right)$, $27.87\left(\mathrm{C}_{2}\right), 25.86\left(\mathrm{C}_{1}\right), 23.68\left(\mathrm{C}_{10}\right), 22.57\left(\mathrm{C}_{7}\right), 14.38\left(\mathrm{C}_{8}\right)$; ESI-MS m/z: $151.17[\mathrm{M}+\mathrm{H}]^{+}$. Anal. Calcd. For $\mathrm{C}_{10} \mathrm{H}_{14} \mathrm{O}$ (150.22): C, 79.96; H, 9.39. Found: C, 79.85; H, 9.36.

\subsection{Synthesis of 3-Caren-5-One Oxime (3)}

3-Caren-5-one (1.5 g, $10 \mathrm{mmol}), \mathrm{NH}_{2} \mathrm{OH} \cdot \mathrm{HCl}(1.04 \mathrm{~g}, 15 \mathrm{mmol})$, and $\mathrm{NaOAc}(4.08 \mathrm{~g}, 30 \mathrm{mmol})$ were dissolved in a mixed solvent $\left(\mathrm{EtOH}: \mathrm{H}_{2} \mathrm{O}=5: 1, v / v\right)$. The reaction mixture was stirred at $80^{\circ} \mathrm{C}$ for $3 \mathrm{~h}$. The reaction process was monitored by TLC. When 3-carene-5-one was fully reacted, the mixture was cooled to room temperature. After the solvent $\mathrm{EtOH}$ was removed by rotary evaporation, the residue was extracted by EtOAc. The separated organic phase was washed with saturated $\mathrm{NaCl}$ solution three times, and concentrated under reduced pressure to obtain the crude product, which was purified by silica gel column chromatography using a mixed solvent $(\mathrm{EtOAc}-$ petroleum ether $=1: 60$, $v / v)$ as eluent.

(Z)-3-Caren-5-one oxime ((Z)-3a) was obtained as a white needle crystal. Yield 63.5\%, m.p.: $96.7-98.1^{\circ} \mathrm{C}$. UV-Vis $(\mathrm{EtOH}) \lambda_{\max }(\log \varepsilon): 239.70(4.28) \mathrm{nm}$; IR $\left(\mathrm{KBr}, \mathrm{cm}^{-1}\right)$ : 3464-3078 (s, br, O-H), 3033 (w, =C-H), $1659(\mathrm{~m}, \mathrm{C}=\mathrm{N}), 1614(\mathrm{~m}) ;{ }^{1} \mathrm{H}-\mathrm{NMR} \delta=9.03(\mathrm{~s}, 1 \mathrm{H}, \mathrm{OH}), \delta 5.87\left(\mathrm{~s}, 1 \mathrm{H}, \mathrm{C}_{4}-\mathrm{H}\right), 2.45(\mathrm{dd}, J=20.0,8.2 \mathrm{~Hz}$, $\left.1 \mathrm{H}, \mathrm{C}_{2}-\mathrm{H}_{\mathrm{a}}\right), 2.12\left(\mathrm{~d}, \mathrm{~J}=20.0 \mathrm{~Hz}, 1 \mathrm{H}, \mathrm{C}_{2}-\mathrm{H}_{\mathrm{b}}\right), 1.97\left(\mathrm{~d}, \mathrm{~J}=8.3 \mathrm{~Hz}, 1 \mathrm{H}, \mathrm{C}_{6}-\mathrm{H}\right), 1.76\left(\mathrm{~s}, 3 \mathrm{H}, \mathrm{C}_{3}-\mathrm{CH}_{3}\right), 1.23$ $\left(\mathrm{s}, 3 \mathrm{H}, \mathrm{C}_{7}-\mathrm{CH}_{3}\right), 1.19\left(\mathrm{t}, J=8.3 \mathrm{~Hz}, 1 \mathrm{H}, \mathrm{C}_{1}-\mathrm{H}\right), 0.89\left(\mathrm{~s}, 3 \mathrm{H}, \mathrm{C}_{7}-\mathrm{CH}_{3}\right) ;{ }^{13} \mathrm{C}-\mathrm{NMR} \delta=155.00\left(\mathrm{C}_{5}\right), 142.27$ $\left(C_{3}\right), 119.32\left(C_{4}\right), 27.97\left(C_{6}\right), 27.08\left(C_{9}\right), 23.39\left(C_{2}\right), 22.37\left(C_{1}\right), 20.71\left(C_{10}\right), 19.67\left(C_{7}\right), 14.59\left(C_{8}\right)$; ESI-MS $m / z: 166.14[\mathrm{M}+\mathrm{H}]^{+}$. Anal. Calcd. For $\mathrm{C}_{10} \mathrm{H}_{15} \mathrm{NO}(165.23): \mathrm{C}, 72.69 ; \mathrm{H}, 9.15 ; \mathrm{N}, 8.48$. Found: $\mathrm{C}, 72.71$; $\mathrm{H}, 9.17 ; \mathrm{N}, 8.49$.

(E)-3-Caren-5-one oxime ((E)-3b) was obtained as a white plate-like crystal. Yield 3\%, m.p.: 73.5-75.0 ${ }^{\circ} \mathrm{C}$. UV-Vis (EtOH) $\lambda_{\max }(\log \varepsilon): 235.80$ (3.89) nm; IR (KBr, cm $\left.{ }^{-1}\right)$ : 3432-3081 (s, br, O-H), 3015 (w, =C-H), $1644(\mathrm{~m}, \mathrm{C}=\mathrm{N}), 1608(\mathrm{~m}) ;{ }^{1} \mathrm{H}-\mathrm{NMR} \delta=8.98(\mathrm{~s}, 1 \mathrm{H}, \mathrm{OH}), 6.64\left(\mathrm{~s}, 1 \mathrm{H}, \mathrm{C}_{4}-\mathrm{H}\right), 2.48(\mathrm{dd}, J=20.4,8.0 \mathrm{~Hz}$, $\left.1 \mathrm{H}, \mathrm{C}_{2}-\mathrm{H}_{\mathrm{a}}\right), 2.16\left(\mathrm{~d}, J=20.5 \mathrm{~Hz}, 1 \mathrm{H}, \mathrm{C}_{2}-\mathrm{H}_{\mathrm{b}}\right), 1.82\left(\mathrm{~s}, 3 \mathrm{H}, \mathrm{C}_{3}-\mathrm{CH}_{3}\right), 1.49\left(\mathrm{~d}, J=8.7 \mathrm{~Hz}, 1 \mathrm{H}, \mathrm{C}_{6}-\mathrm{H}\right), 1.19$ $\left(\mathrm{t}, J=8.3 \mathrm{~Hz}, 1 \mathrm{H}, \mathrm{C}_{1}-\mathrm{H}\right), 1.13\left(\mathrm{~s}, 3 \mathrm{H}, \mathrm{C}_{7}-\mathrm{CH}_{3}\right), 0.88\left(\mathrm{~s}, 3 \mathrm{H}, \mathrm{C}_{7}-\mathrm{CH}_{3}\right) ;{ }^{13} \mathrm{C}-\mathrm{NMR} \delta=152.05\left(\mathrm{C}_{5}\right), 147.32$ $\left(C_{3}\right), 112.59\left(C_{4}\right), 28.03\left(C_{6}\right), 27.71\left(C_{9}\right), 23.86\left(C_{2}\right), 23.54\left(C_{1}\right), 21.82\left(C_{10}\right), 19.61\left(C_{7}\right), 14.15\left(C_{8}\right)$; ESI-MS $m / z: 166.14[\mathrm{M}+\mathrm{H}]^{+}$. Anal. Calcd. For $\mathrm{C}_{10} \mathrm{H}_{15} \mathrm{NO}$ (165.23): $\mathrm{C}, 72.69 ; \mathrm{H}, 9.15 ; \mathrm{N}, 8.48$. Found: $\mathrm{C}, 72.60$; $\mathrm{H}, 9.18 ; \mathrm{N}, 8.46$.

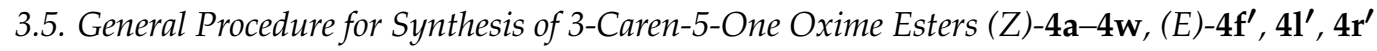

A solution of acyl chloride $(1.2 \mathrm{mmol})$ in $\mathrm{CH}_{2} \mathrm{Cl}_{2}(3 \mathrm{~mL})$ was added slowly to a solution of $(Z)$ or (E)-3-carene-5-one oxime $(1 \mathrm{mmol})$ and triethylamine in $\mathrm{CH}_{2} \mathrm{Cl}_{2}(5 \mathrm{~mL})$ with ice-water cooling. The reaction process was monitored by TLC. Upon completion, $5 \mathrm{~mL}$ deionized water was added to the reaction mixture to destroy the unreacted acyl chloride. Then, the organic layer was separated, washed with deionized water three times, and concentrated in vacuum. The crude product was further

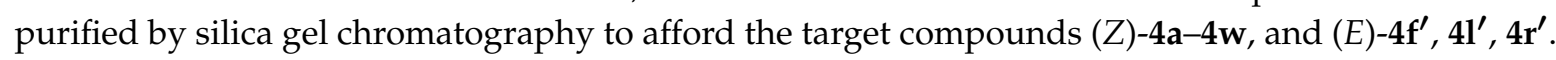




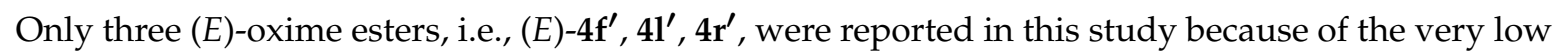
yield of $(E)-3 b$.

(Z)-3-Caren-5-one O-(benzoyl) oxime ((Z)-4a). Pale yellow solid. Yield: $65.4 \%$, m.p.: $97.4-98.7^{\circ} \mathrm{C}$. UV-Vis $(\mathrm{EtOH}) \lambda_{\max }(\log \varepsilon): 257.40(4.34) \mathrm{nm}, 236.70(4.25) \mathrm{nm}$; IR $\left(\mathrm{KBr}, \mathrm{cm}^{-1}\right): 3069(\mathrm{w}), 3015(\mathrm{w}, \mathrm{Ar}-\mathrm{H},=\mathrm{C}-\mathrm{H})$, $1739(\mathrm{~s}, \mathrm{C}=\mathrm{O}), 1658(\mathrm{~m}, \mathrm{C}=\mathrm{N}), 1602(\mathrm{w}), 1581(\mathrm{~s}), 1495(\mathrm{w}), 1456(\mathrm{~m}, \mathrm{Ar}){ }^{1} \mathrm{H}-\mathrm{NMR} \delta=8.10(\mathrm{~d}, J=7.1 \mathrm{~Hz}$, $\left.2 \mathrm{H}, \mathrm{C}_{13}-\mathrm{H}, \mathrm{C}_{17}-\mathrm{H}\right), 7.59\left(\mathrm{t}, J=7.4 \mathrm{~Hz}, 1 \mathrm{H}, \mathrm{C}_{15}-\mathrm{H}\right), 7.47\left(\mathrm{t}, J=7.8 \mathrm{~Hz}, 2 \mathrm{H}, \mathrm{C}_{14}-\mathrm{H}, \mathrm{C}_{16}-\mathrm{H}\right), 6.17(\mathrm{~s}, 1 \mathrm{H}$, $\left.\mathrm{C}_{4}-\mathrm{H}\right), 2.56\left(\mathrm{dd}, J=20.2,8.4 \mathrm{~Hz}, 1 \mathrm{H}, \mathrm{C}_{2}-\mathrm{H}_{\mathrm{a}}\right), 2.21\left(\mathrm{~d}, J=19.5 \mathrm{~Hz}, 1 \mathrm{H}, \mathrm{C}_{2}-\mathrm{H}_{\mathrm{b}}\right), 2.05(\mathrm{~d}, J=8.2 \mathrm{~Hz}, 1 \mathrm{H}$, $\left.\mathrm{C}_{6}-\mathrm{H}\right), 1.85\left(\mathrm{~s}, 3 \mathrm{H}, \mathrm{C}_{3}-\mathrm{CH}_{3}\right), 1.34\left(\mathrm{~d}, J=7.8 \mathrm{~Hz}, 1 \mathrm{H}, \mathrm{C}_{1}-\mathrm{H}\right), 1.30\left(\mathrm{~s}, 3 \mathrm{H}, \mathrm{C}_{7}-\mathrm{CH}_{3}\right), 0.94\left(\mathrm{~s}, 3 \mathrm{H}, \mathrm{C}_{7}-\mathrm{CH}_{3}\right)$; ${ }^{13} \mathrm{C}$-NMR $\delta=164.12\left(C_{11}\right), 161.89\left(C_{5}\right), 147.97\left(C_{3}\right), 133.02\left(C_{15}\right), 129.60\left(C_{12}\right), 129.53\left(C_{13}\right.$ and $\left.C_{17}\right)$, $128.49\left(C_{14}\right.$ and $\left.C_{16}\right), 118.38\left(C_{4}\right), 28.09\left(C_{6}\right), 27.15\left(C_{9}\right), 23.77\left(C_{2}\right), 23.08\left(C_{1}\right), 21.68\left(C_{10}\right), 21.32\left(C_{7}\right)$, $14.59\left(\mathrm{C}_{8}\right)$; ESI-MS m/z: $270.09[\mathrm{M}+\mathrm{H}]^{+}$. Anal. Calcd. For $\mathrm{C}_{17} \mathrm{H}_{19} \mathrm{NO}_{2}$ (269.34): C, 75.81; $\mathrm{H}, 7.11 ; \mathrm{N}$, 5.20. Found: $\mathrm{C}, 75.79 ; \mathrm{H}, 7.12 ; \mathrm{N}, 5.21$.

(Z)-3-Caren-5-one O-(2-chlorobenzoyl) oxime ((Z)-4b). White solid. Yield: $74.0 \%$, m.p.: $116.3-117.8{ }^{\circ} \mathrm{C}$. UV-Vis (EtOH) $\lambda_{\max }(\log \varepsilon): 253.50(4.23) \mathrm{nm}$. IR $\left(\mathrm{KBr}, \mathrm{cm}^{-1}\right)$ : 3048 (w), $3012(\mathrm{w}, \mathrm{Ar}-\mathrm{H},=\mathrm{C}-\mathrm{H}), 1726$ $(\mathrm{s}, \mathrm{C}=\mathrm{O}), 1652(\mathrm{~m}, \mathrm{C}=\mathrm{N}), 1581(\mathrm{~m}), 1464(\mathrm{w}, \mathrm{Ar}) ;{ }^{1} \mathrm{H}-\mathrm{NMR} \delta=7.88\left(\mathrm{dd}, J=7.8,1.6 \mathrm{~Hz}, 1 \mathrm{H}, \mathrm{C}_{17}-\mathrm{H}\right)$, $7.47\left(\mathrm{dd}, J=8.0,1.4 \mathrm{~Hz}, 1 \mathrm{H}, \mathrm{C}_{14}-\mathrm{H}\right), 7.44\left(\mathrm{td}, J=8.1,7.6,1.6 \mathrm{~Hz}, 1 \mathrm{H}, \mathrm{C}_{15}-\mathrm{H}\right), 7.35(\mathrm{td}, J=7.6,1.4 \mathrm{~Hz}$, $\left.1 \mathrm{H}, \mathrm{C}_{16}-\mathrm{H}\right), 6.14\left(\mathrm{~s}, 1 \mathrm{H}, \mathrm{C}_{4}-\mathrm{H}\right), 2.54\left(\mathrm{dd}, J=20.3,8.3 \mathrm{~Hz}, 1 \mathrm{H}, \mathrm{C}_{2}-\mathrm{H}_{\mathrm{a}}\right), 2.21\left(\mathrm{~d}, J=20.3 \mathrm{~Hz}, 1 \mathrm{H}, \mathrm{C}_{2}-\mathrm{H}_{\mathrm{b}}\right)$, $2.08\left(\mathrm{~d}, J=8.1 \mathrm{~Hz}, 1 \mathrm{H}, \mathrm{C}_{6}-\mathrm{H}\right), 1.85\left(\mathrm{~s}, 3 \mathrm{H}, \mathrm{C}_{3}-\mathrm{CH}_{3}\right), 1.29\left(\mathrm{t}, J=8.2 \mathrm{~Hz}, 1 \mathrm{H}, \mathrm{C}_{1}-\mathrm{H}\right), 1.22\left(\mathrm{~s}, 3 \mathrm{H}, \mathrm{C}_{7}-\mathrm{CH}_{3}\right)$, $0.94\left(\mathrm{~s}, 3 \mathrm{H}, \mathrm{C}_{7}-\mathrm{CH}_{3}\right) ;{ }^{13} \mathrm{C}-\mathrm{NMR} \delta=164.06\left(\mathrm{C}_{11}\right), 162.31\left(\mathrm{C}_{5}\right), 148.35\left(\mathrm{C}_{3}\right), 133.30\left(\mathrm{C}_{12}\right), 132.55\left(\mathrm{C}_{15}\right)$, $131.70\left(C_{13}\right), 130.91\left(C_{17}\right), 130.03\left(C_{14}\right), 126.67\left(C_{16}\right), 118.22\left(C_{4}\right), 28.08\left(C_{6}\right), 27.21\left(C_{9}\right), 23.77\left(C_{2}\right), 23.13$ $\left(\mathrm{C}_{1}\right), 21.98\left(\mathrm{C}_{10}\right), 21.43\left(\mathrm{C}_{7}\right), 14.56\left(\mathrm{C}_{8}\right)$; ESI-MS $m / z: 304.07[\mathrm{M}+\mathrm{H}]^{+}$. Anal. Calcd. For $\mathrm{C}_{17} \mathrm{H}_{18} \mathrm{ClNO}_{2}$ (303.78): C, 67.21; H, 5.97; N, 4.61. Found: C, 67.25; H, 5.93; N, 4.62.

(Z)-3-Caren-5-one O-(3-chlorobenzoyl) oxime ((Z)-4c). White solid. Yield: 80.3\%, m.p.: 137.3-138.1 ${ }^{\circ} \mathrm{C}$. UV-Vis (EtOH) $\lambda_{\max }(\log \varepsilon): 259.90(4.08) \mathrm{nm}, 229.80(4.07) \mathrm{nm}$; IR $\left(\mathrm{KBr}, \mathrm{cm}^{-1}\right): 3084(\mathrm{w}), 3024(\mathrm{w}, \mathrm{Ar}-\mathrm{H}$, $=\mathrm{C}-\mathrm{H}), 1741(\mathrm{~s}, \mathrm{C}=\mathrm{O}), 1652(\mathrm{~m}, \mathrm{C}=\mathrm{N}), 1581(\mathrm{~m}), 1491(\mathrm{w}, \mathrm{Ar}){ }^{1} \mathrm{H}-\mathrm{NMR} \delta=8.07\left(\mathrm{~s}, 1 \mathrm{H}, \mathrm{C}_{13}-\mathrm{H}\right), 7.98$ $\left(\mathrm{d}, J=7.8 \mathrm{~Hz}, 1 \mathrm{H}, \mathrm{C}_{17}-\mathrm{H}\right), 7.56\left(\mathrm{~d}, J=8.0 \mathrm{~Hz}, 1 \mathrm{H}, \mathrm{C}_{15}-\mathrm{H}\right), 7.42\left(\mathrm{t}, J=7.9 \mathrm{~Hz}, 1 \mathrm{H}, \mathrm{C}_{16}-\mathrm{H}\right), 6.16(\mathrm{~s}, 1 \mathrm{H}$, $\left.\mathrm{C}_{4}-\mathrm{H}\right), 2.57\left(\mathrm{dd}, J=20.3,8.4 \mathrm{~Hz}, 1 \mathrm{H}, \mathrm{C}_{2}-\mathrm{H}_{\mathrm{a}}\right), 2.22\left(\mathrm{~d}, J=20.3 \mathrm{~Hz}, 1 \mathrm{H}, \mathrm{C}_{2}-\mathrm{H}_{\mathrm{b}}\right), 2.02(\mathrm{~d}, J=8.2 \mathrm{~Hz}, 1 \mathrm{H}$, $\left.\mathrm{C}_{6}-\mathrm{H}\right), 1.86\left(\mathrm{~s}, 3 \mathrm{H}, \mathrm{C}_{3}-\mathrm{CH}_{3}\right), 1.35\left(\mathrm{t}, J=8.4 \mathrm{~Hz}, 1 \mathrm{H}, \mathrm{C}_{1}-\mathrm{H}\right), 1.32\left(\mathrm{~s}, 3 \mathrm{H}, \mathrm{C}_{7}-\mathrm{CH}_{3}\right), 0.94\left(\mathrm{~s}, 3 \mathrm{H}, \mathrm{C}_{7}-\mathrm{CH}_{3}\right)$. ${ }^{13} \mathrm{C}-\mathrm{NMR} \delta=162.94\left(\mathrm{C}_{11}\right), 162.32\left(\mathrm{C}_{5}\right), 148.54\left(\mathrm{C}_{3}\right), 134.66\left(\mathrm{C}_{12}\right), 133.07\left(\mathrm{C}_{15}\right), 131.31\left(\mathrm{C}_{14}\right), 129.85\left(\mathrm{C}_{16}\right)$, $129.65\left(C_{13}\right), 127.62\left(C_{17}\right), 118.12\left(C_{4}\right), 28.03\left(C_{6}\right), 27.16\left(C_{9}\right), 23.80\left(C_{2}\right), 23.11\left(C_{1}\right), 21.80\left(C_{10}\right), 21.35$ $\left(\mathrm{C}_{7}\right), 14.59\left(\mathrm{C}_{8}\right)$; ESI-MS $m / z: 304.05[\mathrm{M}+\mathrm{H}]^{+}$. Anal. Calcd. For $\mathrm{C}_{17} \mathrm{H}_{18} \mathrm{ClNO}_{2}$ (303.78): $\mathrm{C}, 67.21 ; \mathrm{H}$, 5.97; N, 4.61. Found: C, 67.23; H, 5.98; N, 4.60.

(Z)-3-Caren-5-one O-(4-chlorobenzoyl) oxime ((Z)-4d). White solid. Yield: 78.6\%, m.p.: 149.9-151.3 ${ }^{\circ} \mathrm{C}$. UV-Vis (EtOH) $\lambda_{\max }(\log \varepsilon): 258.80(4.32) \mathrm{nm}, 245.90$ (4.32) nm; IR (KBr, cm $\left.{ }^{-1}\right): 3066$ (w), 3024 (w, Ar-H, $=\mathrm{C}-\mathrm{H}), 1736(\mathrm{~s}, \mathrm{C}=\mathrm{O}), 1659(\mathrm{~m}, \mathrm{C}=\mathrm{N}), 1596(\mathrm{~m}), 1578(\mathrm{~m}), 1486(\mathrm{~m}, \mathrm{Ar}){ }^{1} \mathrm{H}-\mathrm{NMR} \delta=8.03(\mathrm{~d}, J=8.4 \mathrm{~Hz}$, $\left.2 \mathrm{H}, \mathrm{C}_{13}-\mathrm{H}, \mathrm{C}_{17}-\mathrm{H}\right), 7.45\left(\mathrm{~d}, J=8.4 \mathrm{~Hz}, 2 \mathrm{H}, \mathrm{C}_{14}-\mathrm{H}, \mathrm{C}_{16}-\mathrm{H}\right), 6.16\left(\mathrm{~s}, 1 \mathrm{H}, \mathrm{C}_{4}-\mathrm{H}\right), 2.56(\mathrm{dd}, J=20.2,8.3 \mathrm{~Hz}$, $\left.1 \mathrm{H}, \mathrm{C}_{2}-\mathrm{H}_{\mathrm{a}}\right), 2.22\left(\mathrm{~d}, J=20.3 \mathrm{~Hz}, 1 \mathrm{H}, \mathrm{C}_{2}-\mathrm{H}_{\mathrm{b}}\right), 2.01\left(\mathrm{~d}, J=8.1 \mathrm{~Hz}, 1 \mathrm{H}, \mathrm{C}_{6}-\mathrm{H}\right), 1.85\left(\mathrm{~s}, 3 \mathrm{H}, \mathrm{C}_{3}-\mathrm{CH}_{3}\right), 1.34$ $\left(\mathrm{t}, J=8.2 \mathrm{~Hz}, 1 \mathrm{H}, \mathrm{C}_{1}-\mathrm{H}\right), 1.29\left(\mathrm{~s}, 3 \mathrm{H}, \mathrm{C}_{7}-\mathrm{CH}_{3}\right), 0.94\left(\mathrm{~s}, 3 \mathrm{H}, \mathrm{C}_{7}-\mathrm{CH}_{3}\right) ;{ }^{13} \mathrm{C}-\mathrm{NMR} \delta=163.34\left(\mathrm{C}_{11}\right), 162.15$ $\left(C_{5}\right), 148.32\left(C_{3}\right), 139.52\left(C_{15}\right), 130.88\left(C_{12}\right), 128.89\left(C_{13}, C_{14}\right), 128.02\left(C_{15}\right), 118.22\left(C_{3}\right), 28.10\left(C_{6}\right), 27.15$ $\left(C_{9}\right), 23.79\left(C_{2}\right), 23.13\left(C_{1}\right), 21.77\left(C_{10}\right), 21.32\left(C_{7}\right), 14.58\left(C_{8}\right)$; ESI-MS $m / z: 304.04\left[\mathrm{M}+\mathrm{H}^{+}\right.$. Anal. Calcd. For $\mathrm{C}_{17} \mathrm{H}_{18} \mathrm{ClNO}_{2}$ (303.78): C, 67.21; H, 5.97; N, 4.61. Found: $\mathrm{C}, 67.23 ; \mathrm{H}, 5.95 ; \mathrm{N}, 4.63$.

(Z)-3-Caren-5-one O-(2-fluorobenzoyl) oxime ((Z)-4e). White solid. Yield: 76.9\%, m.p.: 128.7-131.2 ${ }^{\circ} \mathrm{C}$. UV-Vis (EtOH) $\lambda_{\max }(\log \varepsilon): 257.60(4.29) \mathrm{nm}, 224.70$ (4.29) nm; IR (KBr, cm $\left.{ }^{-1}\right)$ : 3057 (w), 3009 (w, Ar-H, $=\mathrm{C}-\mathrm{H}), 1736(\mathrm{~s}, \mathrm{C}=\mathrm{O}), 1650(\mathrm{~m}, \mathrm{C}=\mathrm{N}), 1608(\mathrm{~m}), 1575(\mathrm{~m}), 1486(\mathrm{~m}), 1453(\mathrm{~m}, \mathrm{Ar}) ;{ }^{1} \mathrm{H}-\mathrm{NMR} \delta=8.07$ $\left(\mathrm{td}, J=7.5,1.8 \mathrm{~Hz}, 1 \mathrm{H}, \mathrm{C}_{17}-\mathrm{H}\right), 7.57-7.52\left(\mathrm{~m}, 1 \mathrm{H}, \mathrm{C}_{15}-\mathrm{H}\right), 7.25\left(\mathrm{td}, J=7.7,1.1 \mathrm{~Hz}, 1 \mathrm{H}, \mathrm{C}_{16}-\mathrm{H}\right), 7.16$ (ddd, $\left.J=10.7,8.3,0.9 \mathrm{~Hz}, 1 \mathrm{H}, \mathrm{C}_{14}-\mathrm{H}\right), 6.15\left(\mathrm{~s}, 1 \mathrm{H}, \mathrm{C}_{4}-\mathrm{H}\right), 2.55\left(\mathrm{dd}, J=20.3,8.4 \mathrm{~Hz}, 1 \mathrm{H}, \mathrm{C}_{2}-\mathrm{H}_{\mathrm{a}}\right), 2.21$ $\left(\mathrm{d}, J=20.3 \mathrm{~Hz}, 1 \mathrm{H}, \mathrm{C}_{2}-\mathrm{H}_{\mathrm{b}}\right), 2.12\left(\mathrm{~d}, J=8.1 \mathrm{~Hz}, 1 \mathrm{H}, \mathrm{C}_{6}-\mathrm{H}\right), 1.85\left(\mathrm{~s}, 3 \mathrm{H}, \mathrm{C}_{3}-\mathrm{CH}_{3}\right), 1.31(\mathrm{t}, J=7.9 \mathrm{~Hz}, 1 \mathrm{H}$, $\left.\mathrm{C}_{1}-\mathrm{H}\right), 1.26\left(\mathrm{~s}, 3 \mathrm{H}, \mathrm{C}_{7}-\mathrm{CH}_{3}\right), 0.93\left(\mathrm{~s}, 3 \mathrm{H}, \mathrm{C}_{7}-\mathrm{CH}_{3}\right) .{ }^{13} \mathrm{C}-\mathrm{NMR} \delta=162.29\left(\mathrm{C}_{11}\right), 160.83\left(\mathrm{C}_{5}\right), 148.32\left(\mathrm{C}_{3}\right)$, 
$134.61\left(\mathrm{C}_{15}\right), 134.55\left(\mathrm{C}_{17}\right), 132.63\left(\mathrm{C}_{16}\right), 124.13\left(\mathrm{C}_{12}\right), 118.23\left(\mathrm{C}_{4}\right), 117.02\left(\mathrm{C}_{13}\right), 116.87\left(\mathrm{C}_{14}\right), 27.90\left(\mathrm{C}_{6}\right)$, $27.24\left(\mathrm{C}_{9}\right), 23.78\left(\mathrm{C}_{2}\right), 23.13\left(\mathrm{C}_{1}\right), 21.88\left(\mathrm{C}_{10}\right), 21.45\left(\mathrm{C}_{7}\right), 14.56\left(\mathrm{C}_{8}\right)$; ESI-MS m/z: $288.08[\mathrm{M}+\mathrm{H}]^{+}$. Anal. Calcd. For $\mathrm{C}_{17} \mathrm{H}_{18} \mathrm{FNO}_{2}$ (287.33): C, 71.06; H, 6.31; N, 4.87. Found: C, 71.10; H, 6.30; N, 4.85.

(Z)-3-Caren-5-one O-(4-fluorobenzoyl) oxime ((Z)-4f). White solid. Yield: $65.2 \%$, m.p.: 133.2-135.0 ${ }^{\circ} \mathrm{C}$. UV-Vis (EtOH) $\lambda_{\max }(\log \varepsilon): 257.00(4.29) \mathrm{nm}, 229.10(4.26) \mathrm{nm}$; IR $\left(\mathrm{KBr}, \mathrm{cm}^{-1}\right): 3041$ (w), 3018 (w, Ar-H, $=\mathrm{C}-\mathrm{H}), 1748(\mathrm{~s}, \mathrm{C}=\mathrm{O}), 1659(\mathrm{~m}, \mathrm{C}=\mathrm{N}), 1602(\mathrm{~m}), 1581(\mathrm{~m}), 1507(\mathrm{~m}, \mathrm{Ar}){ }^{1} \mathrm{H}-\mathrm{NMR}$ 8: $8.11(\mathrm{dd}, J=8.9$, $\left.5.4 \mathrm{~Hz}, 2 \mathrm{H}, \mathrm{C}_{13}-\mathrm{H}, \mathrm{C}_{17}-\mathrm{H}\right), 7.15\left(\mathrm{t}, J=8.7 \mathrm{~Hz}, 2 \mathrm{H}, \mathrm{C}_{14}-\mathrm{H}, \mathrm{C}_{16}-\mathrm{H}\right), 6.16\left(\mathrm{~s}, 1 \mathrm{H}, \mathrm{C}_{4}-\mathrm{H}\right), 2.56(\mathrm{dd}, J=20.3$, $\left.8.4 \mathrm{~Hz}, 1 \mathrm{H}, \mathrm{C}_{2}-\mathrm{H}_{\mathrm{a}}\right), 2.22\left(\mathrm{~d}, J=20.3 \mathrm{~Hz}, 1 \mathrm{H}, \mathrm{C}_{2}-\mathrm{H}_{\mathrm{b}}\right), 2.02\left(\mathrm{~d}, J=8.2 \mathrm{~Hz}, 1 \mathrm{H}, \mathrm{C}_{6}-\mathrm{H}\right), 1.85\left(\mathrm{~s}, 3 \mathrm{H}, \mathrm{C}_{3}-\mathrm{CH}_{3}\right)$, $1.34\left(\mathrm{t}, J=8.2 \mathrm{~Hz}, 1 \mathrm{H}, \mathrm{C}_{1}-\mathrm{H}\right), 1.30\left(\mathrm{~s}, 3 \mathrm{H}, \mathrm{C}_{7}-\mathrm{CH}_{3}\right), 0.94\left(\mathrm{~s}, 3 \mathrm{H}, \mathrm{C}_{7}-\mathrm{CH}_{3}\right) ;{ }^{13} \mathrm{C}-\mathrm{NMR} \delta: 166.63\left(\mathrm{C}_{11}\right)$, $164.95\left(C_{5}\right), 163.23\left(C_{15}\right), 162.01\left(C_{12}\right), 148.19\left(C_{3}\right), 132.07\left(C_{13}\right.$ or $\left.C_{17}\right), 132.01\left(C_{13}\right.$ or $\left.C_{17}\right), 118.27\left(C_{4}\right)$, $115.79\left(\mathrm{C}_{14}\right.$ or $\left.\mathrm{C}_{16}\right), 115.64\left(\mathrm{C}_{14}\right.$ or $\left.\mathrm{C}_{16}\right), 28.11\left(\mathrm{C}_{6}\right), 27.15\left(\mathrm{C}_{9}\right), 23.78\left(\mathrm{C}_{2}\right), 23.12\left(\mathrm{C}_{1}\right), 21.73\left(\mathrm{C}_{10}\right), 21.32$ $\left(\mathrm{C}_{7}\right), 14.59\left(\mathrm{C}_{8}\right)$; ESI-MS $m / z: 288.07[\mathrm{M}+\mathrm{H}]^{+}$. Anal. Calcd. For $\mathrm{C}_{17} \mathrm{H}_{18} \mathrm{FNO}_{2}$ (287.33): C, 71.06; $\mathrm{H}$, 6.31; N, 4.87. Found: $\mathrm{C}, 71.03 ; \mathrm{H}, 6.29 ; \mathrm{N}, 4.86$.

(Z)-3-Caren-5-one O-(2-methoxybenzoyl) oxime ((Z)-4g). White solid. Yield: $65.0 \%$, m.p.: $123.9-125.5^{\circ} \mathrm{C}$. UV-Vis (EtOH) $\lambda_{\max }(\log \varepsilon): 254.50$ (4.38) nm; IR (KBr): 3054 (w), 3006 (w, Ar-H, =C-H), 1724 (s, C=O), $1656(\mathrm{~m}, \mathrm{C}=\mathrm{N}), 1599(\mathrm{~m}), 1581(\mathrm{~m}), 1489(\mathrm{~m}), 1462(\mathrm{~m}, \mathrm{Ar}){ }^{1} \mathrm{H}-\mathrm{NMR} \delta=7.85$ (dd, J = 7.7, $1.8 \mathrm{~Hz}, 1 \mathrm{H}$, $\left.\mathrm{C}_{17}-\mathrm{H}\right), 7.48\left(\mathrm{td}, J=9.0,8.2,1.8 \mathrm{~Hz}, 1 \mathrm{H}, \mathrm{C}_{15}-\mathrm{H}\right), 7.04-6.99\left(\mathrm{~m}, 1 \mathrm{H}, \mathrm{C}_{16}-\mathrm{H}\right), 7.01-6.97\left(\mathrm{~m}, 1 \mathrm{H}, \mathrm{C}_{14}-\mathrm{H}\right)$, $6.15\left(\mathrm{~s}, 1 \mathrm{H}, \mathrm{C}_{4}-\mathrm{H}\right), 3.89\left(\mathrm{~s}, 3 \mathrm{H}, \mathrm{O}-\mathrm{CH}_{3}\right), 2.53\left(\mathrm{dd}, J=20.2,8.3 \mathrm{~Hz}, 1 \mathrm{H}, \mathrm{C}_{2}-\mathrm{H}_{\mathrm{a}}\right), 2.20(\mathrm{~d}, J=20.2 \mathrm{~Hz}, 1 \mathrm{H}$, $\left.\mathrm{C}_{2}-\mathrm{H}_{\mathrm{b}}\right), 2.08\left(\mathrm{~d}, J=8.1 \mathrm{~Hz}, 1 \mathrm{H}, \mathrm{C}_{6}-\mathrm{H}\right), 1.84\left(\mathrm{~s}, 3 \mathrm{H}, \mathrm{C}_{3}-\mathrm{CH}_{3}\right), 1.29\left(\mathrm{t}, J=8.1 \mathrm{~Hz}, 1 \mathrm{H}, \mathrm{C}_{6}-\mathrm{H}\right), 1.23(\mathrm{~s}, 3 \mathrm{H}$, $\left.\mathrm{C}_{7}-\mathrm{CH}_{3}\right), 0.93\left(\mathrm{~s}, 3 \mathrm{H}, \mathrm{C}_{7}-\mathrm{CH}_{3}\right) ;{ }^{13} \mathrm{C}-\mathrm{NMR} \delta=164.58\left(\mathrm{C}_{11}\right), 161.72\left(\mathrm{C}_{5}\right), 158.98\left(\mathrm{C}_{13}\right), 147.53\left(\mathrm{C}_{3}\right), 133.45$ $\left(\mathrm{C}_{15}\right), 131.79\left(\mathrm{C}_{17}\right), 120.20\left(\mathrm{C}_{12}\right), 119.68\left(\mathrm{C}_{16}\right), 118.58\left(\mathrm{C}_{4}\right), 111.97\left(\mathrm{C}_{14}\right), 55.94\left(\mathrm{C}_{18}\right), 28.17\left(\mathrm{C}_{6}\right), 27.21\left(\mathrm{C}_{9}\right)$, $23.73\left(\mathrm{C}_{2}\right), 23.21\left(\mathrm{C}_{1}\right), 21.70\left(\mathrm{C}_{10}\right), 21.30\left(\mathrm{C}_{7}\right), 14.56\left(\mathrm{C}_{8}\right)$; ESI-MS m/z: $300.01[\mathrm{M}+\mathrm{H}]^{+}$. Anal. Calcd. For $\mathrm{C}_{17} \mathrm{H}_{21} \mathrm{NO}_{2}$ (299.36): C, 72.22; H, 7.07; N, 4.68. Found: $\mathrm{C}, 72.17 ; \mathrm{H}, 7.04 ; \mathrm{N}, 4.71$.

(Z)-3-Caren-5-one O-(2-methylbenzoyl) oxime ((Z)-4h). White solid. Yield: 65\%, m.p.: 64.7-67.5 ${ }^{\circ} \mathrm{C}$. UV-Vis $(\mathrm{EtOH}) \lambda_{\max }(\log \varepsilon): 255.80(4.42) \mathrm{nm}$; IR $\left(\mathrm{KBr}, \mathrm{cm}^{-1}\right): 3048(\mathrm{w}), 3015(\mathrm{w}, \mathrm{Ar}-\mathrm{H},=\mathrm{C}-\mathrm{H}), 1748$ (s, C=O), $1653(\mathrm{~m}, \mathrm{C}=\mathrm{N}), 1605(\mathrm{w}), 1581(\mathrm{~m}), 1486(\mathrm{w}), 1453(\mathrm{~m}, \mathrm{Ar}) ;{ }^{1} \mathrm{H}-\mathrm{NMR} \delta=7.92\left(\mathrm{~d}, J=6.8 \mathrm{~Hz}, 1 \mathrm{H}, \mathrm{C}_{17}-\mathrm{H}\right)$, $7.42\left(\mathrm{t}, J=7.5 \mathrm{~Hz}, 1 \mathrm{H}, \mathrm{C}_{15}-\mathrm{H}\right), 7.28\left(\mathrm{~d}, J=7.6 \mathrm{~Hz}, 1 \mathrm{H}, \mathrm{C}_{16}-\mathrm{H}\right), 7.26\left(\mathrm{~d}, J=7.5 \mathrm{~Hz}, 1 \mathrm{H}, \mathrm{C}_{14}-\mathrm{H}\right), 6.16(\mathrm{~s}, 1 \mathrm{H}$, $\left.\mathrm{C}_{4}-\mathrm{H}\right), 2.66\left(\mathrm{~s}, 3 \mathrm{H}, \mathrm{C}_{13}-\mathrm{CH}_{3}\right), 2.54\left(\mathrm{dd}, J=20.6,8.7 \mathrm{~Hz}, 1 \mathrm{H}, \mathrm{C}_{2}-\mathrm{H}_{\mathrm{a}}\right), 2.20\left(\mathrm{~d}, J=20.3 \mathrm{~Hz}, 1 \mathrm{H}, \mathrm{C}_{2}-\mathrm{H}_{\mathrm{b}}\right), 2.00$ $\left(\mathrm{d}, J=8.2 \mathrm{~Hz}, 1 \mathrm{H}, \mathrm{C}_{6}-\mathrm{H}\right), 1.85\left(\mathrm{~s}, 3 \mathrm{H}, \mathrm{C}_{3}-\mathrm{CH}_{3}\right), 1.30\left(\mathrm{t}, J=8.2 \mathrm{~Hz}, 1 \mathrm{H}, \mathrm{C}_{1}-\mathrm{H}\right), 1.25\left(\mathrm{~s}, 3 \mathrm{H}, \mathrm{C}_{7}-\mathrm{CH}_{3}\right), 0.94$ $\left(\mathrm{s}, 3 \mathrm{H}, \mathrm{C}_{7}-\mathrm{CH}_{3}\right) ;{ }^{13} \mathrm{C}-\mathrm{NMR} \delta=165.43\left(\mathrm{C}_{11}\right), 161.56\left(\mathrm{C}_{5}\right), 147.75\left(\mathrm{C}_{3}\right), 140.06\left(\mathrm{C}_{13}\right), 131.95\left(\mathrm{C}_{15}\right), 131.67$ $\left(\mathrm{C}_{12}\right), 130.30\left(\mathrm{C}_{17}\right), 129.17\left(\mathrm{C}_{14}\right), 125.70\left(\mathrm{C}_{16}\right), 118.49\left(\mathrm{C}_{4}\right), 28.08\left(\mathrm{C}_{6}\right), 27.13\left(\mathrm{C}_{9}\right), 23.74\left(\mathrm{C}_{2}\right), 23.02\left(\mathrm{C}_{1}\right)$, $21.72\left(\mathrm{C}_{10}\right.$ and $\left.\mathrm{C}_{18}\right), 21.41\left(\mathrm{C}_{7}\right), 14.61\left(\mathrm{C}_{8}\right)$; ESI-MS $m / z: 284.12[\mathrm{M}+\mathrm{H}]^{+}$. Anal. Calcd. For $\mathrm{C}_{18} \mathrm{H}_{21} \mathrm{NO}_{2}$ (283.36): C, 76.29; H, 7.47; N, 4.94. Found: C, 76.21; H, 7.50; N, 4.90.

(Z)-3-Caren-5-one O-(3-methylbenzoyl) oxime ((Z)-4i). White solid. Yield: 70.0\%, m.p.: 131.8-133.2 ${ }^{\circ} \mathrm{C}$. UV-Vis $(\mathrm{EtOH}) \lambda_{\max }(\log \varepsilon): 258.30(4.31) \mathrm{nm}$; IR $\left(\mathrm{KBr}, \mathrm{cm}^{-1}\right)$ : $3054(\mathrm{w}), 3021$ (w, Ar-H, =C-H), 1736 $(\mathrm{s}, \mathrm{C}=\mathrm{O}), 1650(\mathrm{~m}, \mathrm{C}=\mathrm{N}), 1572(\mathrm{~m}), 1453(\mathrm{~m}, \mathrm{Ar}) ;{ }^{1} \mathrm{H}-\mathrm{NMR} \delta=7.93\left(\mathrm{~s}, 1 \mathrm{H}, \mathrm{C}_{13}-\mathrm{H}\right), 7.89(\mathrm{~d}, J=7.6 \mathrm{~Hz}$, $\left.1 \mathrm{H}, \mathrm{C}_{17}-\mathrm{H}\right), 7.39\left(\mathrm{~d}, J=7.6 \mathrm{~Hz}, 1 \mathrm{H}, \mathrm{C}_{15}-\mathrm{H}\right), 7.35\left(\mathrm{t}, J=7.6 \mathrm{~Hz}, 1 \mathrm{H}, \mathrm{C}_{16}-\mathrm{H}\right), 6.16\left(\mathrm{~s}, 1 \mathrm{H}, \mathrm{C}_{4}-\mathrm{H}\right), 2.56(\mathrm{dd}$, $\left.J=19.9,8.1 \mathrm{~Hz}, 1 \mathrm{H}, \mathrm{C}_{2}-\mathrm{H}_{\mathrm{a}}\right), 2.41\left(\mathrm{~s}, 3 \mathrm{H}, \mathrm{C}_{14}-\mathrm{CH}_{3}\right), 2.21\left(\mathrm{~d}, J=20.3 \mathrm{~Hz}, 1 \mathrm{H}, \mathrm{C}_{2}-\mathrm{H}_{\mathrm{b}}\right), 2.05(\mathrm{~d}, J=8.2 \mathrm{~Hz}$, $\left.1 \mathrm{H}, \mathrm{C}_{6}-\mathrm{H}\right), 1.85\left(\mathrm{~s}, 3 \mathrm{H}, \mathrm{C}_{3}-\mathrm{CH}_{3}\right), 1.33\left(\mathrm{t}, J=8.3 \mathrm{~Hz}, 1 \mathrm{H}, \mathrm{C}_{1}-\mathrm{H}\right), 1.31\left(\mathrm{~s}, 3 \mathrm{H}, \mathrm{C}_{7}-\mathrm{CH}_{3}\right), 0.94\left(\mathrm{~s}, 3 \mathrm{H}, \mathrm{C}_{7}-\mathrm{CH}_{3}\right)$; ${ }^{13} \mathrm{C}-\mathrm{NMR} \delta=164.29\left(\mathrm{C}_{11}\right), 161.83\left(\mathrm{C}_{5}\right), 147.93\left(\mathrm{C}_{3}\right), 138.29\left(\mathrm{C}_{14}\right), 133.80\left(\mathrm{C}_{15}\right), 130.22\left(\mathrm{C}_{12}\right), 129.48\left(\mathrm{C}_{13}\right)$, $128.36\left(\mathrm{C}_{16}\right), 126.58\left(\mathrm{C}_{17}\right), 118.39\left(\mathrm{C}_{4}\right), 28.03\left(\mathrm{C}_{6}\right), 27.15\left(\mathrm{C}_{9}\right), 23.78\left(\mathrm{C}_{2}\right), 23.02\left(\mathrm{C}_{1}\right), 21.62\left(\mathrm{C}_{18}\right), 21.35$ $\left(\mathrm{C}_{10}\right), 21.31\left(\mathrm{C}_{7}\right), 14.60\left(\mathrm{C}_{8}\right)$; ESI-MS $m / z: 284.13[\mathrm{M}+\mathrm{H}]^{+}$. Anal. Calcd. For $\mathrm{C}_{18} \mathrm{H}_{21} \mathrm{NO}_{2}$ (283.36): $\mathrm{C}$, 76.29; H, 7.47; N, 4.94. Found: C, 76.25; H, 7.49; N, 4.92.

(Z)-3-Caren-5-one O-(4-methylbenzoyl) oxime ((Z)-4j). White solid. Yield: 75.3\%, m.p.: $125.9-127.6{ }^{\circ} \mathrm{C}$. UV-Vis $(\mathrm{EtOH}) \lambda_{\max }(\log \varepsilon): 259.30(4.38) \mathrm{nm} ; \mathrm{IR}\left(\mathrm{KBr}, \mathrm{cm}^{-1}\right): 3066(\mathrm{w}), 3033(\mathrm{w}, \mathrm{Ar}-\mathrm{H},=\mathrm{C}-\mathrm{H}), 1742$ $(\mathrm{s}, \mathrm{C}=\mathrm{O}), 1656(\mathrm{~m}, \mathrm{C}=\mathrm{N}), 1617(\mathrm{~m}), 1572(\mathrm{~m}), 1510(\mathrm{~m}), 1450(\mathrm{~m}, \mathrm{Ar}) ;{ }^{1} \mathrm{H}-\mathrm{NMR} \delta=7.99(\mathrm{~d}, J=8.2 \mathrm{~Hz}$, $\left.2 \mathrm{H}, \mathrm{C}_{13}-\mathrm{H}, \mathrm{C}_{17}-\mathrm{H}\right), 7.27\left(\mathrm{~d}, J=6.5 \mathrm{~Hz}, 2 \mathrm{H}, \mathrm{C}_{14}-\mathrm{H}, \mathrm{C}_{16}-\mathrm{H}\right), 6.16\left(\mathrm{~s}, 1 \mathrm{H}, \mathrm{C}_{4}-\mathrm{H}\right), 2.55(\mathrm{dd}, J=20.2,7.7 \mathrm{~Hz}$, $\left.1 \mathrm{H}, \mathrm{C}_{2}-\mathrm{H}_{\mathrm{a}}\right), 2.42\left(\mathrm{~s}, 3 \mathrm{H}, \mathrm{C}_{15}-\mathrm{CH}_{3}\right), 2.21\left(\mathrm{~d}, J=20.3 \mathrm{~Hz}, 1 \mathrm{H}, \mathrm{C}_{2}-\mathrm{H}_{\mathrm{b}}\right), 2.04\left(\mathrm{~d}, J=8.2 \mathrm{~Hz}, 1 \mathrm{H}, \mathrm{C}_{6}-\mathrm{H}\right), 1.84$ 
$\left(\mathrm{s}, 3 \mathrm{H}, \mathrm{C}_{3}-\mathrm{CH}_{3}\right), 1.32\left(\mathrm{t}, J=8.0 \mathrm{~Hz}, 1 \mathrm{H}, \mathrm{C}_{1}-\mathrm{H}\right), 1.29\left(\mathrm{~s}, 3 \mathrm{H}, \mathrm{C}_{7}-\mathrm{CH}_{3}\right), 0.94\left(\mathrm{~s}, 3 \mathrm{H}, \mathrm{C}_{7}-\mathrm{CH}_{3}\right) .{ }^{13} \mathrm{C}-\mathrm{NMR}$ $\delta=164.23\left(C_{11}\right), 161.72\left(C_{5}\right), 147.80\left(C_{3}\right), 143.75\left(C_{15}\right), 129.57\left(C_{12}\right), 129.21\left(C_{13}\right.$ and $\left.C_{17}\right), 126.78\left(C_{14}\right.$ and $\left.\mathrm{C}_{16}\right), 118.44\left(\mathrm{C}_{4}\right), 28.07\left(\mathrm{C}_{6}\right), 27.15\left(\mathrm{C}_{9}\right), 23.76\left(\mathrm{C}_{2}\right), 23.05\left(\mathrm{C}_{1}\right), 21.70\left(\mathrm{C}_{18}\right), 21.63\left(\mathrm{C}_{10}\right), 21.32\left(\mathrm{C}_{7}\right)$, $14.59\left(\mathrm{C}_{8}\right)$; ESI-MS $m / z: 284.17$ [M + H] $]^{+}$. Anal. Calcd. For $\mathrm{C}_{18} \mathrm{H}_{21} \mathrm{NO}_{2}$ (283.36): C, 76.29; $\mathrm{H}, 7.47 ; \mathrm{N}$, 4.94. Found: $\mathrm{C}, 76.32 ; \mathrm{H}, 7.45 ; \mathrm{N}, 4.93$.

(Z)-3-Caren-5-one O-(2,4-dichlorobenzoyl) oxime ((Z)-4k). White solid. Yield: 65.0\%, m.p.: 82.0-83.0 ${ }^{\circ} \mathrm{C}$. UV-Vis (EtOH) $\lambda_{\max }(\log \varepsilon): 249.50(4.30) \mathrm{nm}$; IR $\left(\mathrm{KBr}, \mathrm{cm}^{-1}\right): 3087(\mathrm{w}), 3018(\mathrm{w}, \mathrm{Ar}-\mathrm{H},=\mathrm{C}-\mathrm{H}), 1736(\mathrm{~s}$, $\mathrm{C}=\mathrm{O}), 1653(\mathrm{~m}, \mathrm{C}=\mathrm{N}), 1587(\mathrm{~m}), 1545(\mathrm{~m}), 1468(\mathrm{~m}), 1450(\mathrm{~m}, \mathrm{Ar}){ }^{1} \mathrm{H}-\mathrm{NMR} \delta=7.85(\mathrm{~d}, J=8.4 \mathrm{~Hz}, 1 \mathrm{H}$, $\left.\mathrm{C}_{17}-\mathrm{H}\right), 7.50\left(\mathrm{~d}, J=2.0 \mathrm{~Hz}, 1 \mathrm{H}, \mathrm{C}_{14}-\mathrm{H}\right), 7.34\left(\mathrm{dd}, J=8.4,2.0 \mathrm{~Hz}, 1 \mathrm{H}, \mathrm{C}_{16}\right), 6.13\left(\mathrm{~s}, 1 \mathrm{H}, \mathrm{C}_{4}-\mathrm{H}\right), 2.55(\mathrm{dd}$, $\left.J=20.6,8.7 \mathrm{~Hz}, 1 \mathrm{H}, \mathrm{C}_{2}-\mathrm{H}_{\mathrm{a}}\right), 2.21\left(\mathrm{~d}, J=20.3 \mathrm{~Hz}, 1 \mathrm{H}, \mathrm{C}_{2}-\mathrm{H}_{\mathrm{b}}\right), 2.05\left(\mathrm{~d}, J=8.1 \mathrm{~Hz}, 1 \mathrm{H}, \mathrm{C}_{6}-\mathrm{H}\right), 1.85(\mathrm{~s}, 3 \mathrm{H}$, $\left.\mathrm{C}_{3}-\mathrm{CH}_{3}\right), 1.30\left(\mathrm{t}, J=8.0 \mathrm{~Hz}, 1 \mathrm{H}, \mathrm{C}_{1}-\mathrm{H}\right), 1.22\left(\mathrm{~s}, 3 \mathrm{H}, \mathrm{C}_{7}-\mathrm{CH}_{3}\right), 0.93\left(\mathrm{~s}, 3 \mathrm{H}, \mathrm{C}_{7}-\mathrm{CH}_{3}\right) .{ }^{13} \mathrm{C}-\mathrm{NMR} \delta=163.20$ $\left(C_{11}\right), 162.44\left(C_{5}\right), 148.59\left(C_{3}\right), 138.35\left(C_{15}\right), 134.38\left(C_{13}\right), 132.79\left(C_{12}\right), 130.86\left({ }_{17}\right), 128.34\left({ }_{14}\right), 127.16$ $\left(C_{16}\right), 118.10\left(C_{4}\right), 28.10\left(C_{6}\right), 27.20\left(C_{9}\right), 23.78\left(C_{2}\right), 23.16\left(C_{1}\right), 22.04\left(C_{10}\right), 21.48\left(C_{7}\right), 14.56\left(C_{8}\right)$; ESI-MS $m / z: 338.06[\mathrm{M}+\mathrm{H}]^{+}$. Anal. Calcd. For $\mathrm{C}_{17} \mathrm{H}_{17} \mathrm{Cl}_{2} \mathrm{NO}_{2}$ (338.23): $\mathrm{C}, 60.37 ; \mathrm{H}, 5.07 ; \mathrm{N}, 4.14$. Found: $\mathrm{C}$, 60.30; H, 5.11; N, 4.17.

(Z)-3-Caren-5-one O-(2,3-dichlorobenzoyl) oxime ((Z)-4l). Yellow liquid. Yield: 60.7\%. UV-Vis (EtOH) $\lambda_{\max }(\log \varepsilon): 254.19(4.16) \mathrm{nm}, 224.42(4.28) \mathrm{nm}$; IR (thin film, $\left.\mathrm{cm}^{-1}\right): 3076(\mathrm{w}), 3014(\mathrm{w}, \mathrm{Ar}-\mathrm{H},=\mathrm{C}-\mathrm{H})$, $1750(\mathrm{~s}, \mathrm{C}=\mathrm{O}), 1653(\mathrm{~m}, \mathrm{C}=\mathrm{N}), 1581(\mathrm{~m}), 1560(\mathrm{w}), 1516(\mathrm{w}), 1447(\mathrm{~m}, \mathrm{Ar}) ;{ }^{1} \mathrm{H}-\mathrm{NMR} \delta=7.71(\mathrm{dd}, J=7.8$, $\left.1.6 \mathrm{~Hz}, 1 \mathrm{H}, \mathrm{C}_{17}-\mathrm{H}\right), 7.62\left(\mathrm{dd}, J=8.0,1.6 \mathrm{~Hz}, 1 \mathrm{H}, \mathrm{C}_{15}-\mathrm{H}\right), 7.30\left(\mathrm{t}, J=7.9 \mathrm{~Hz}, 1 \mathrm{H}, \mathrm{C}_{16}-\mathrm{H}\right), 6.13(\mathrm{~s}, 1 \mathrm{H}$, $\left.\mathrm{C}_{4}-\mathrm{H}\right), 2.55\left(\mathrm{dd}, J=21.1,8.3 \mathrm{~Hz}, 1 \mathrm{H}, \mathrm{C}_{2}-\mathrm{H}_{\mathrm{a}}\right), 2.21\left(\mathrm{~d}, J=21.1 \mathrm{~Hz}, 1 \mathrm{H}, \mathrm{C}_{2}-\mathrm{H}_{\mathrm{b}}\right), 2.02(\mathrm{~d}, J=8.1 \mathrm{~Hz}, 1 \mathrm{H}$, $\left.\mathrm{C}_{6}-\mathrm{H}\right), 1.85\left(\mathrm{~s}, 3 \mathrm{H}, \mathrm{C}_{3}-\mathrm{CH}_{3}\right), 1.30\left(\mathrm{t}, J=8.0 \mathrm{~Hz}, 1 \mathrm{H}, \mathrm{C}_{1}-\mathrm{H}\right), 1.22\left(\mathrm{~s}, 3 \mathrm{H}, \mathrm{C}_{7}-\mathrm{CH}_{3}\right), 0.94\left(\mathrm{~s}, 3 \mathrm{H}, \mathrm{C}_{7}-\mathrm{CH}_{3}\right)$. ${ }^{13} \mathrm{C}-\mathrm{NMR} \delta=164.70\left(\mathrm{C}_{11}\right), 163.64\left(\mathrm{C}_{5}\right), 149.74\left(\mathrm{C}_{3}\right), 135.48\left(\mathrm{C}_{12}\right), 134.17\left(\mathrm{C}_{15}\right), 133.69\left(\mathrm{C}_{14}\right), 132.38\left(\mathrm{C}_{13}\right)$, $130.37\left(C_{17}\right), 128.32\left(C_{16}\right), 119.05\left(C_{4}\right), 29.11\left(C_{6}\right), 28.21\left(C_{9}\right), 24.78\left(C_{2}\right), 24.20\left(C_{1}\right), 23.08(10), 22.36\left(C_{7}\right)$, 15.56 (8); ESI-MS $m / z: 337.99$ [M - H] $]^{-}$. Anal. Calcd. For $\mathrm{C}_{17} \mathrm{H}_{17} \mathrm{Cl}_{2} \mathrm{NO}_{2}$ (338.23): $\mathrm{C}, 60.37 ; \mathrm{H}, 5.07 ; \mathrm{N}$, 4.14. Found: $\mathrm{C}, 60.40 ; \mathrm{H}, 5.09 ; \mathrm{N}, 4.15$.

(Z)-3-Caren-5-one O-(4-chloromethylbenzoyl) oxime ((Z)-4m). White solid. Yield: 78\%, m.p.: $121.9-123.2{ }^{\circ} \mathrm{C}$. UV-Vis (EtOH) $\lambda_{\max }(\log \varepsilon): 258.00(4.23) \mathrm{nm}, 238.50$ (4.31) nm; IR (KBr, cm $\left.{ }^{-1}\right)$ : 3039 (w), 3009 (w, Ar-H, =C-H), $1748(\mathrm{~s}, \mathrm{C}=\mathrm{O}), 1653(\mathrm{~m}, \mathrm{C}=\mathrm{N}), 1611(\mathrm{~m}), 1575(\mathrm{~m}), 1516(\mathrm{w}), 1426(\mathrm{~m}, \mathrm{Ar}){ }^{1} \mathrm{H}-\mathrm{NMR} \delta=8.09$ $\left(\mathrm{d}, J=8.3 \mathrm{~Hz}, 2 \mathrm{H}, \mathrm{C}_{13}-\mathrm{H}, \mathrm{C}_{17}-\mathrm{H}\right), 7.50\left(\mathrm{~d}, J=8.2 \mathrm{~Hz}, 2 \mathrm{H}, \mathrm{C}_{14}-\mathrm{H}, \mathrm{C}_{16}-\mathrm{H}\right), 6.16\left(\mathrm{~s}, 1 \mathrm{H}, \mathrm{C}_{4}-\mathrm{H}\right), 4.63(\mathrm{~s}, 2 \mathrm{H}$, $\left.\mathrm{C}_{18}-\mathrm{H}\right), 2.56\left(\mathrm{dd}, J=20.3,8.3 \mathrm{~Hz}, 1 \mathrm{H}, \mathrm{C}_{2}-\mathrm{H}_{\mathrm{a}}\right), 2.22\left(\mathrm{~d}, J=20.3 \mathrm{~Hz}, 1 \mathrm{H}, \mathrm{C}_{2}-\mathrm{H}_{\mathrm{b}}\right), 2.03(\mathrm{~d}, J=8.1 \mathrm{~Hz}, 1 \mathrm{H}$, $\left.\mathrm{C}_{6}-\mathrm{H}\right), 1.85\left(\mathrm{~s}, 3 \mathrm{H}, \mathrm{C}_{3}-\mathrm{CH}_{3}\right), 1.34\left(\mathrm{t}, J=8.2 \mathrm{~Hz}, 1 \mathrm{H}, \mathrm{C}_{1}-\mathrm{H}\right), 1.30\left(\mathrm{~s}, 3 \mathrm{H}, \mathrm{C}_{7}-\mathrm{CH}_{3}\right), 0.94\left(\mathrm{~s}, 3 \mathrm{H}, \mathrm{C}_{7}-\mathrm{CH}_{3}\right)$; ${ }^{13} \mathrm{C}-\mathrm{NMR} \delta=163.66\left(\mathrm{C}_{11}\right), 162.08\left(\mathrm{C}_{5}\right), 148.22\left(\mathrm{C}_{3}\right), 142.39\left(\mathrm{C}_{15}\right), 130.63\left(\mathrm{C}_{12}\right), 129.97\left(\mathrm{C}_{13}, \mathrm{C}_{17}\right), 128.62$ $\left(\mathrm{C}_{14}, \mathrm{C}_{16}\right), 118.27\left(\mathrm{C}_{4}\right), 45.36\left(\mathrm{C}_{18}\right), 28.10\left(\mathrm{C}_{6}\right), 27.16\left(\mathrm{C}_{9}\right), 23.78\left(\mathrm{C}_{2}\right), 23.12\left(\mathrm{C}_{1}\right), 21.75\left(\mathrm{C}_{10}\right), 21.34\left(\mathrm{C}_{7}\right)$, $14.59\left(\mathrm{C}_{8}\right)$; ESI-MS m/z: $318.10[\mathrm{M}+\mathrm{H}]^{+}$. Anal. Calcd. For $\mathrm{C}_{18} \mathrm{H}_{20} \mathrm{ClNO}_{2}(317.81): \mathrm{C}, 68.03 ; \mathrm{H}, 6.34 ; \mathrm{N}$, 4.41. Found: $\mathrm{C}, 68.00 ; \mathrm{H}, 6.35 ; \mathrm{N}, 4.43$.

(Z)-3-Caren-5-one O-cyclopentylcarbonyl oxime ((Z)-4n). White solid. Yield: $64.5 \%$, m.p.: $58.0-60.2{ }^{\circ} \mathrm{C}$. UV-Vis (EtOH) $\lambda_{\max }(\log \varepsilon): 241.40(4.19) \mathrm{nm}$; IR $\left(\mathrm{KBr}, \mathrm{cm}^{-1}\right): 3030(\mathrm{w},=\mathrm{C}-\mathrm{H}), 1757(\mathrm{~s}, \mathrm{C}=\mathrm{O}), 1653$ $(\mathrm{m}, \mathrm{C}=\mathrm{N}) ;{ }^{1} \mathrm{H}-\mathrm{NMR} \delta=6.07\left(\mathrm{~s}, 1 \mathrm{H}, \mathrm{C}_{4}-\mathrm{H}\right), 2.89\left(\mathrm{p}, J=8.0 \mathrm{~Hz}, 1 \mathrm{H}, \mathrm{C}_{12}-\mathrm{H}\right), 2.51(\mathrm{dd}, J=20.3,8.4 \mathrm{~Hz}, 1 \mathrm{H}$, $\left.\mathrm{C}_{2}-\mathrm{H}_{\mathrm{a}}\right), 2.17\left(\mathrm{~d}, J=20.3 \mathrm{~Hz}, 1 \mathrm{H}, \mathrm{C}_{2}-\mathrm{H}_{\mathrm{b}}\right), 1.94\left(\mathrm{tt}, J=12.5,6.8 \mathrm{~Hz}, 4 \mathrm{H}, \mathrm{C}_{13}-\mathrm{H}_{2}, \mathrm{C}_{16}-\mathrm{H}_{2}\right), 1.89(\mathrm{~d}, J=8.2 \mathrm{~Hz}$, $\left.1 \mathrm{H}, \mathrm{C}_{6}-\mathrm{H}\right), 1.81\left(\mathrm{~s}, 3 \mathrm{H}, \mathrm{C}_{3}-\mathrm{CH}_{3}\right), 1.78-1.72\left(\mathrm{~m}, 2 \mathrm{H}, \mathrm{C}_{14}-2 \mathrm{H}\right), 1.62\left(\mathrm{dt}, J=8.9,4.2 \mathrm{~Hz}, 2 \mathrm{H}, \mathrm{C}_{15}-\mathrm{H}\right), 1.27$ $\left(\mathrm{t}, J=8.3 \mathrm{~Hz}, 1 \mathrm{H}, \mathrm{C}_{1}-\mathrm{H}\right), 1.23\left(\mathrm{~s}, 3 \mathrm{H}, \mathrm{C}_{7}-\mathrm{CH}_{3}\right), 0.89\left(\mathrm{~s}, 3 \mathrm{H}, \mathrm{C}_{7}-\mathrm{CH}_{3}\right) .{ }^{13} \mathrm{C}-\mathrm{NMR} \delta=174.14\left(\mathrm{C}_{11}\right), 161.27$ $\left(C_{5}\right), 147.49\left(C_{3}\right), 118.48\left(C_{4}\right), 42.99\left(C_{12}\right), 30.08\left(C_{13}\right), 30.02\left(C_{16}\right), 28.02\left(C_{6}\right), 27.12\left(C_{9}\right), 25.86\left(C_{14}\right.$ and $\left.\mathrm{C}_{15}\right), 23.71\left(\mathrm{C}_{2}\right), 22.97\left(\mathrm{C}_{1}\right), 21.50\left(\mathrm{C}_{10}\right), 21.04\left(\mathrm{C}_{7}\right), 14.56\left(\mathrm{C}_{8}\right)$; ESI-MS $m / z: 262.13[\mathrm{M}+\mathrm{H}]^{+}$. Anal. Calcd. For $\mathrm{C}_{16} \mathrm{H}_{23} \mathrm{NO}_{2}$ (261.36): C, 73.53; $\mathrm{H}, 8.87 ; \mathrm{N}$, 5.36. Found: $\mathrm{C}, 73.51 ; \mathrm{H}, 8.89 ; \mathrm{N}, 5.37$.

(Z)-3-Caren-5-one O-cyclohexylcarbonyl oxime ((Z)-4o). White solid. Yield: $64.8 \%$, m.p.: 81.4-82.5 ${ }^{\circ} \mathrm{C}$. UV-Vis (EtOH) $\lambda_{\max }(\log \varepsilon): 243.60$ (4.29) nm: IR $\left(\mathrm{KBr}, \mathrm{cm}^{-1}\right): 3027$ (w, =C-H), $1753(\mathrm{~s}, \mathrm{C}=\mathrm{O}), 1650(\mathrm{~m}$, $\mathrm{C}=\mathrm{N}) ;{ }^{1} \mathrm{H}-\mathrm{NMR} \delta=6.07\left(\mathrm{~s}, 1 \mathrm{H}, \mathrm{C}_{4}-\mathrm{H}\right), 2.54-2.44\left(\mathrm{~m}, 2 \mathrm{H}, \mathrm{C}_{2}-\mathrm{H}_{\mathrm{a}}, \mathrm{C}_{12}-\mathrm{H}\right), 2.17\left(\mathrm{~d}, J=20.3 \mathrm{~Hz}, 1 \mathrm{H}, \mathrm{C}_{2}-\mathrm{H}_{\mathrm{b}}\right)$, $1.98\left(\mathrm{~s}, 2 \mathrm{H}, \mathrm{C}_{13}-\mathrm{H}\right), 1.89\left(\mathrm{~d}, J=8.2 \mathrm{~Hz}, 1 \mathrm{H}, \mathrm{C}_{6}-\mathrm{H}\right), 1.81\left(\mathrm{~s}, 3 \mathrm{H}, \mathrm{C}_{3}-\mathrm{CH}_{3}\right), 1.78(\mathrm{dd}, J=9.1,3.9 \mathrm{~Hz}, 2 \mathrm{H}$, 
$\left.\mathrm{C}_{17}-\mathrm{H}\right), 1.57\left(\mathrm{q}, J=11.7 \mathrm{~Hz}, 2 \mathrm{H}, \mathrm{C}_{15}-\mathrm{H}\right), 1.37-1.25\left(\mathrm{~m}, 5 \mathrm{H}, \mathrm{C}_{1}-\mathrm{H}, \mathrm{C}_{14}-\mathrm{H}, \mathrm{C}_{16}-\mathrm{H}\right), 1.23\left(\mathrm{~s}, 3 \mathrm{H}, \mathrm{C}_{7}-\mathrm{CH}_{3}\right)$, $0.89\left(\mathrm{~s}, 3 \mathrm{H}, \mathrm{C}_{7}-\mathrm{CH}_{3}\right) ;{ }^{13} \mathrm{C}-\mathrm{NMR} \delta=173.30\left(\mathrm{C}_{11}\right), 161.43\left(\mathrm{C}_{5}\right), 147.52\left(\mathrm{C}_{3}\right), 118.47\left(\mathrm{C}_{4}\right), 42.54\left(\mathrm{C}_{12}\right), 29.10$ $\left(C_{13}\right), 29.03\left(C_{17}\right), 28.03\left(C_{6}\right), 27.11\left(C_{9}\right), 25.77\left(C_{14}\right), 25.48\left(C_{15}\right), 25.43\left(C_{16}\right), 23.70\left(C_{2}\right), 22.97\left(C_{1}\right), 21.50$ $\left(\mathrm{C}_{10}\right), 21.04\left(\mathrm{C}_{7}\right), 14.55\left(\mathrm{C}_{8}\right)$; ESI-MS $m / z: 276.22[\mathrm{M}+\mathrm{H}]^{+}$. Anal. Calcd. For $\mathrm{C}_{17} \mathrm{H}_{25} \mathrm{NO}_{2}$ (275.39): $\mathrm{C}$, 74.14; H, 9.15; N, 5.09. Found: C, 74.16; H, 9.12; N, 5.06.

(Z)-3-caren-5-one O- $\alpha$-furylcarbonyl oxime ((Z)-4p). White solid. Yield: $82.0 \%$, m.p.: $114.7-115.6{ }^{\circ} \mathrm{C}$. UV-Vis (EtOH) $\lambda_{\max }(\log \varepsilon): 268.90(4.28) \mathrm{nm}$; IR $\left(\mathrm{KBr}, \mathrm{cm}^{-1}\right): 3110(\mathrm{~m}), 3006(\mathrm{w},=\mathrm{C}-\mathrm{H}), 1754$ (s, C=O), $1653(\mathrm{~m}, \mathrm{C}=\mathrm{N}) ;{ }^{1} \mathrm{H}-\mathrm{NMR} \delta=7.65-7.61\left(\mathrm{~m}, 1 \mathrm{H}, \mathrm{C}_{15}-\mathrm{H}\right), 7.22\left(\mathrm{~d}, J=3.5 \mathrm{~Hz}, 1 \mathrm{H}, \mathrm{C}_{13}-\mathrm{H}\right), 6.55(\mathrm{dd}, J=3.5$, $\left.1.7 \mathrm{~Hz}, 1 \mathrm{H}, \mathrm{C}_{14}-\mathrm{H}\right), 6.13\left(\mathrm{~s}, 1 \mathrm{H}, \mathrm{C}_{4}-\mathrm{H}\right), 2.55\left(\mathrm{dd}, J=20.3,8.3 \mathrm{~Hz}, 1 \mathrm{H}, \mathrm{C}_{2}-\mathrm{H}_{\mathrm{a}}\right), 2.21(\mathrm{~d}, J=20.3 \mathrm{~Hz}, 1 \mathrm{H}$, $\left.\mathrm{C}_{2}-\mathrm{H}_{\mathrm{b}}\right), 2.02\left(\mathrm{~d}, J=8.1 \mathrm{~Hz}, 1 \mathrm{H}, \mathrm{C}_{6}-\mathrm{H}\right), 1.84\left(\mathrm{~s}, 3 \mathrm{H}, \mathrm{C}_{3}-\mathrm{CH}_{3}\right), 1.33\left(\mathrm{t}, J=8.1 \mathrm{~Hz}, 1 \mathrm{H}, \mathrm{C}_{1}-\mathrm{H}\right), 1.28(\mathrm{~s}, 4 \mathrm{H}$, $\left.\mathrm{C}_{7}-\mathrm{CH}_{3}\right), 0.93\left(\mathrm{~s}, 3 \mathrm{H}, \mathrm{C}_{7}-\mathrm{CH}_{3}\right) .{ }^{13} \mathrm{C}-\mathrm{NMR} \delta=162.13\left(\mathrm{C}_{11}\right), 156.70\left(\mathrm{C}_{5}\right), 148.25\left(\mathrm{C}_{3}\right), 146.63\left(\mathrm{C}_{12}\right), 143.61$ $\left(C_{15}\right), 118.14\left(C_{4}\right), 117.95\left(C_{13}\right), 111.84\left(C_{14}\right), 28.00\left(C_{6}\right), 27.19\left(C_{9}\right), 23.77\left(C_{2}\right), 23.15\left(C_{1}\right), 21.78\left(C_{10}\right)$, $21.22\left(\mathrm{C}_{7}\right), 14.54\left(\mathrm{C}_{8}\right)$; ESI-MS m/z: $260.07[\mathrm{M}+\mathrm{H}]^{+}$. Anal. Calcd. For $\mathrm{C}_{15} \mathrm{H}_{17} \mathrm{NO}_{3}(259.30): \mathrm{C}, 69.48 ; \mathrm{H}$, 6.61; N, 5.40. Found: C, 69.50; H, 6.58; N, 5.41 .

(Z)-3-Caren-5-one O- $\alpha$-thienylcarbonyl oxime ((Z)-4q). White solid. Yield: $75.5 \%$, m.p.: $108.4-109.3{ }^{\circ} \mathrm{C}$. UV-Vis (EtOH) $\lambda_{\max }(\log \varepsilon): 278.80(4.30) \mathrm{nm}, 253.90(4.36) \mathrm{nm}$; IR $\left(\mathrm{KBr}, \mathrm{cm}^{-1}\right): 3116(\mathrm{w}), 3018(\mathrm{w},=\mathrm{C}-\mathrm{H})$, $1739(\mathrm{~s}, \mathrm{C}=\mathrm{O}), 1650(\mathrm{~m}, \mathrm{C}=\mathrm{N}) ;{ }^{1} \mathrm{H}-\mathrm{NMR} \delta=7.91\left(\mathrm{dd}, J=3.8,1.2 \mathrm{~Hz}, 1 \mathrm{H}, \mathrm{C}_{13}-\mathrm{H}\right), 7.59(\mathrm{dd}, J=5.0$, $\left.1.2 \mathrm{~Hz}, 1 \mathrm{H}, \mathrm{C}_{15}-\mathrm{H}\right), 7.15\left(\mathrm{dd}, J=4.9,3.8 \mathrm{~Hz}, 1 \mathrm{H}, \mathrm{C}_{14}-\mathrm{H}\right), 6.14\left(\mathrm{~s}, 1 \mathrm{H}, \mathrm{C}_{4}-\mathrm{H}\right), 2.55(\mathrm{dd}, J=20.6,8.7 \mathrm{~Hz}$, $\left.1 \mathrm{H}, \mathrm{C}_{2}-\mathrm{H}_{\mathrm{a}}\right), 2.21\left(\mathrm{~d}, J=21.1 \mathrm{~Hz}, 1 \mathrm{H}, \mathrm{C}_{2}-\mathrm{H}_{\mathrm{b}}\right), 2.03\left(\mathrm{~d}, J=8.1 \mathrm{~Hz}, 1 \mathrm{H}, \mathrm{C}_{6}-\mathrm{H}\right), 1.85\left(\mathrm{~s}, 3 \mathrm{H}, \mathrm{C}_{3}-\mathrm{CH}_{3}\right), 1.33$ $\left(\mathrm{d}, J=8.1 \mathrm{~Hz}, 1 \mathrm{H}, \mathrm{C}_{6}-\mathrm{H}\right), 1.31\left(\mathrm{~s}, 3 \mathrm{H}, \mathrm{C}_{7}-\mathrm{CH}_{3}\right), 0.93\left(\mathrm{~s}, 3 \mathrm{H}, \mathrm{C}_{7}-\mathrm{CH}_{3}\right) .{ }^{13} \mathrm{C}-\mathrm{NMR} \delta=161.82\left(\mathrm{C}_{11}\right), 159.90$ $\left(C_{5}\right), 148.19\left(C_{3}\right), 133.81\left(C_{12}\right), 132.45\left(C_{13}\right), 132.18\left(C_{15}\right), 127.84\left(C_{14}\right), 118.15\left(C_{4}\right), 28.00\left(C_{6}\right), 27.16\left(C_{9}\right)$, $23.78\left(\mathrm{C}_{2}\right), 23.03\left(\mathrm{C}_{1}\right), 21.70\left(\mathrm{C}_{10}\right), 21.29\left(\mathrm{C}_{7}\right), 14.57\left(\mathrm{C}_{8}\right)$; ESI-MS $\mathrm{m} / z: 276.06[\mathrm{M}+\mathrm{H}]^{+}$. Anal. Calcd. For $\mathrm{C}_{15} \mathrm{H}_{17} \mathrm{NO}_{2} \mathrm{~S}$ (275.37): $\mathrm{C}, 65.43 ; \mathrm{H}, 6.22 ; \mathrm{N}, 5.09$. Found: $\mathrm{C}, 65.45 ; \mathrm{H}, 6.21 ; \mathrm{N}, 5.10$.

(Z)-3-Caren-5-one O- $\beta$-pyridylcarbonyl oxime ((Z)-4r). Brown solid. Yield: 80.0\%, m.p.: $97.0-98.7{ }^{\circ} \mathrm{C}$. UV-Vis (EtOH) $\lambda_{\max }(\log \varepsilon): 262.60(4.18) \mathrm{nm}, 223.80(4.14) \mathrm{nm} ; \mathrm{IR}\left(\mathrm{KBr}, \mathrm{cm}^{-1}\right): 3065(\mathrm{w}), 3039(\mathrm{w},=\mathrm{C}-\mathrm{H})$, $1743(\mathrm{~s}, \mathrm{C}=\mathrm{O}), 1655(\mathrm{~m}, \mathrm{C}=\mathrm{N}) ;{ }^{1} \mathrm{H}-\mathrm{NMR} \delta=9.28\left(\mathrm{~s}, 1 \mathrm{H}, \mathrm{C}_{13}-\mathrm{H}\right), 8.81\left(\mathrm{~d}, J=4.8 \mathrm{~Hz}, 1 \mathrm{H}, \mathrm{C}_{14}-\mathrm{H}\right), 8.38(\mathrm{~d}$, $\left.J=7.9 \mathrm{~Hz}, 1 \mathrm{H}, \mathrm{C}_{16}-\mathrm{H}\right), 7.45\left(\mathrm{dd}, J=7.9,4.9 \mathrm{~Hz}, 1 \mathrm{H}, \mathrm{C}_{15}-\mathrm{H}\right), 6.16\left(\mathrm{~s}, 1 \mathrm{H}, \mathrm{C}_{4}-\mathrm{H}\right), 2.57(\mathrm{dd}, J=20.3,8.4 \mathrm{~Hz}$, $\left.1 \mathrm{H}, \mathrm{C}_{2}-\mathrm{H}_{\mathrm{a}}\right), 2.23\left(\mathrm{~d}, \mathrm{~J}=20.3 \mathrm{~Hz}, 1 \mathrm{H}, \mathrm{C}_{2}-\mathrm{H}_{\mathrm{b}}\right), 2.03\left(\mathrm{~d}, J=8.1 \mathrm{~Hz}, 1 \mathrm{H}, \mathrm{C}_{6}-\mathrm{H}\right), 1.86\left(\mathrm{~s}, 3 \mathrm{H}, \mathrm{C}_{3}-\mathrm{CH}_{3}\right), 1.36$ $\left(\mathrm{t}, J=8.3 \mathrm{~Hz}, 1 \mathrm{H}, \mathrm{C}_{1}-\mathrm{H}\right), 1.31\left(\mathrm{~s}, 3 \mathrm{H}, \mathrm{C}_{7}-\mathrm{CH}_{3}\right), 0.95\left(\mathrm{~s}, 3 \mathrm{H}, \mathrm{C}_{7}-\mathrm{CH}_{3}\right) .{ }^{13} \mathrm{C}-\mathrm{NMR} \delta=162.88\left(\mathrm{C}_{11}\right), 162.48$ $\left(\mathrm{C}_{5}\right), 153.47\left(\mathrm{C}_{3}\right), 150.53\left(\mathrm{C}_{14}\right), 148.73\left(\mathrm{C}_{13}\right), 137.20\left(\mathrm{C}_{16}\right), 125.72\left(\mathrm{C}_{12}\right), 123.55\left(\mathrm{C}_{15}\right), 118.06\left(\mathrm{C}_{4}\right), 28.15$ $\left(\mathrm{C}_{6}\right), 27.18\left(\mathrm{C}_{9}\right), 23.81\left(\mathrm{C}_{2}\right), 23.21\left(\mathrm{C}_{6}\right), 21.94\left(\mathrm{C}_{10}\right), 21.34\left(\mathrm{C}_{7}\right), 14.58\left(\mathrm{C}_{8}\right)$; ESI-MS $m / z: 271.14[\mathrm{M}+\mathrm{H}]^{+}$. Anal. Calcd. For $\mathrm{C}_{16} \mathrm{H}_{18} \mathrm{~N}_{2} \mathrm{O}_{2}$ (270.33): $\mathrm{C}, 71.09 ; \mathrm{H}, 6.71 ; \mathrm{N}, 10.36$. Found: $\mathrm{C}, 71.06 ; \mathrm{H}, 6.70 ; \mathrm{N}, 10.38$.

(Z)-3-Caren-5-one O-(2-chloropyridylcarbonyl) oxime ((Z)-4s). Brown solid. Yield: $82.4 \%$, m.p.: 107.1-109.4 ${ }^{\circ} \mathrm{C}$. UV-Vis $(\mathrm{EtOH}) \lambda_{\max }(\log \varepsilon): 257.50(4.28) \mathrm{nm}, 229.10(4.15) \mathrm{nm}$; IR $\left(\mathrm{KBr}, \mathrm{cm}^{-1}\right)$ : $3051(\mathrm{w}), 3018(\mathrm{w},=\mathrm{C}-\mathrm{H}), 1736(\mathrm{~s}, \mathrm{C}=\mathrm{O}), 1650(\mathrm{~m}, \mathrm{C}=\mathrm{N}) ;{ }^{1} \mathrm{H}-\mathrm{NMR} \delta=8.55(\mathrm{dd}, J=4.8,2.0 \mathrm{~Hz}, 1 \mathrm{H}$, $\left.\mathrm{C}_{14}-\mathrm{H}\right), 8.23\left(\mathrm{dd}, J=7.7,2.0 \mathrm{~Hz}, 1 \mathrm{H}, \mathrm{C}_{16}-\mathrm{H}\right), 7.38\left(\mathrm{dd}, J=7.7,4.8 \mathrm{~Hz}, 1 \mathrm{H}, \mathrm{C}_{15}-\mathrm{H}\right), 6.13\left(\mathrm{~s}, 1 \mathrm{H}, \mathrm{C}_{4}-\mathrm{H}\right), 2.56$ $\left(\mathrm{dd}, J=20.7,8.7 \mathrm{~Hz}, 1 \mathrm{H}, \mathrm{C}_{2}-\mathrm{H}_{\mathrm{a}}\right), 2.22\left(\mathrm{~d}, J=21.1 \mathrm{~Hz}, 1 \mathrm{H}, \mathrm{C}_{2}-\mathrm{H}_{\mathrm{b}}\right), 2.08\left(\mathrm{~d}, J=8.1 \mathrm{~Hz}, 1 \mathrm{H}, \mathrm{C}_{6}-\mathrm{H}\right), 1.86$ $\left(\mathrm{s}, 3 \mathrm{H}, \mathrm{C}_{3}-\mathrm{CH}_{3}\right), 1.32\left(\mathrm{t}, J=8.0 \mathrm{~Hz}, 1 \mathrm{H}, \mathrm{C}_{1}-\mathrm{H}\right), 1.24\left(\mathrm{~s}, 3 \mathrm{H}, \mathrm{C}_{7}-\mathrm{CH}_{3}\right), 0.94\left(\mathrm{~s}, 3 \mathrm{H}, \mathrm{C}_{7}-\mathrm{CH}_{3}\right) .{ }^{13} \mathrm{C}-\mathrm{NMR}$ $\delta=163.05\left(C_{11}\right), 162.81\left(C_{5}\right), 151.91\left(C_{3}\right), 149.48\left(C_{14}\right), 149.00\left(C_{13}\right), 140.74\left(C_{16}\right), 126.97\left(C_{12}\right), 122.24$ $\left(C_{15}\right), 117.92\left(C_{4}\right), 28.11\left(C_{6}\right), 27.23\left(C_{9}\right), 23.80\left(C_{2}\right), 23.23\left(C_{6}\right), 22.20\left(C_{10}\right), 21.46\left(C_{7}\right), 14.56\left(C_{8}\right)$; ESI-MS $m / z: 305.03[\mathrm{M}+\mathrm{H}]^{+}$. Anal. Calcd. For $\mathrm{C}_{16} \mathrm{H}_{17} \mathrm{ClN}_{2} \mathrm{O}_{2}$ (304.77): $\mathrm{C}, 63.05 ; \mathrm{H}, 5.62 ; \mathrm{N}, 9.19$. Found: $\mathrm{C}$, 63.01; H, 5.63; N, 9.21.

(Z)-3-Caren-5-one O-n-hexanoyl oxime ((Z)-4t). Yellow liquid. Yield: $63.5 \%$. UV-vis $(\mathrm{EtOH}) \lambda_{\max }(\log \varepsilon)$ : $241.80(4.23) \mathrm{nm}$; IR (thin film, $\left.\mathrm{cm}^{-1}\right)$ : $1766(\mathrm{~s}, \mathrm{C}=\mathrm{O}), 1659(\mathrm{~m}, \mathrm{C}=\mathrm{N}), 763(\mathrm{~m}), 751\left(\mathrm{~m},-\left(\mathrm{CH}_{2}\right)_{n^{-}}, n>4\right)$; ${ }^{1} \mathrm{H}-\mathrm{NMR} \delta=6.06\left(\mathrm{~s}, 1 \mathrm{H}, \mathrm{C}_{4}-\mathrm{H}\right), 2.51\left(\mathrm{dd}, J=20.3,8.3 \mathrm{~Hz}, 1 \mathrm{H}, \mathrm{C}_{2}-\mathrm{H}_{\mathrm{a}}\right), 2.45\left(\mathrm{t}, J=7.5 \mathrm{~Hz}, 2 \mathrm{H}, \mathrm{C}_{12}-\mathrm{H}\right), 2.18$ $\left(\mathrm{d}, J=20.3 \mathrm{~Hz}, 1 \mathrm{H}, \mathrm{C}_{2}-\mathrm{H}_{\mathrm{b}}\right), 1.90\left(\mathrm{~d}, J=8.2 \mathrm{~Hz}, 1 \mathrm{H}, \mathrm{C}_{6}-\mathrm{H}\right), 1.82\left(\mathrm{~s}, 3 \mathrm{H}, \mathrm{C}_{4}-\mathrm{CH}_{3}\right), 1.72(\mathrm{q}, J=7.4 \mathrm{~Hz}, 2 \mathrm{H}$, $\left.\mathrm{C}_{13}-\mathrm{H}\right), 1.36\left(\mathrm{dt}, J=7.1,4.4 \mathrm{~Hz}, 4 \mathrm{H}, \mathrm{C}_{14}-\mathrm{H}, \mathrm{C}_{15}-\mathrm{H}\right), 1.28\left(\mathrm{~d}, J=8.6 \mathrm{~Hz}, 1 \mathrm{H}, \mathrm{C}_{1}-\mathrm{H}\right), 1.23\left(\mathrm{~s}, 3 \mathrm{H}, \mathrm{C}_{7}-\mathrm{CH}_{3}\right)$, $0.91\left(\mathrm{~d}, J=7.1 \mathrm{~Hz}, 3 \mathrm{H}, \mathrm{C}_{16}-\mathrm{H}\right), 0.89\left(\mathrm{~s}, 3 \mathrm{H}, \mathrm{C}_{7}-\mathrm{CH}_{3}\right) .{ }^{13} \mathrm{C}-\mathrm{NMR} \delta=171.52\left(\mathrm{C}_{11}\right), 161.20\left(\mathrm{C}_{5}\right), 147.60$ 
$\left(C_{3}\right), 118.45\left(C_{4}\right), 33.33\left(C_{12}\right), 31.32\left(C_{13}\right), 28.02\left(C_{6}\right), 27.13\left(C_{9}\right), 24.76\left(C_{14}\right), 23.70\left(C_{2}\right), 23.03\left(C_{1}\right), 22.31$ $\left(\mathrm{C}_{7}\right), 21.59\left(\mathrm{C}_{10}\right), 21.00\left(\mathrm{C}_{15}\right), 14.53\left(\mathrm{C}_{8}\right), 13.91\left(\mathrm{C}_{16}\right)$; ESI-MS m/z: $264.20[\mathrm{M}+\mathrm{H}]^{+}$. Anal. Calcd. For $\mathrm{C}_{16} \mathrm{H}_{25} \mathrm{NO}_{2}$ (263.38): C, 72.96; H, 9.57; N, 5.32. Found: $\mathrm{C}, 72.92 ; \mathrm{H}, 9.60 ; \mathrm{N}, 5.31$.

(Z)-3-Caren-5-one O-n-propionyl oxime ((Z)-4u). Yellow liquid. Yield: $66.3 \%$. UV-Vis (EtOH) $\lambda_{\max }(\log \varepsilon)$ : $242.00(4.35) \mathrm{nm}$; IR (thin film, $\left.\mathrm{cm}^{-1}\right)$ : $1765(\mathrm{~s}, \mathrm{C}=\mathrm{N}), 1655(\mathrm{~m}, \mathrm{C}=\mathrm{N}) ;{ }^{1} \mathrm{H}-\mathrm{NMR} \delta=6.06\left(\mathrm{~s}, 1 \mathrm{H}, \mathrm{C}_{4}-\mathrm{H}\right)$, 2.55-2.47 (m, 3H, $\left.\mathrm{C}_{2}-\mathrm{H}_{\mathrm{a}}, \mathrm{C}_{12}-\mathrm{H}\right), 2.18\left(\mathrm{~d}, J=20.3 \mathrm{~Hz}, 1 \mathrm{H}, \mathrm{C}_{2}-\mathrm{H}_{\mathrm{b}}\right), 1.90\left(\mathrm{~d}, J=8.2 \mathrm{~Hz}, 1 \mathrm{H}, \mathrm{C}_{6}-\mathrm{H}\right), 1.82$ $\left(\mathrm{s}, 3 \mathrm{H}, \mathrm{C}_{3}-\mathrm{CH}_{3}\right), 1.29-1.24\left(\mathrm{~m}, 4 \mathrm{H}, \mathrm{C}_{1}-\mathrm{H}, \mathrm{C}_{13}-3 \mathrm{H}\right), 1.23\left(\mathrm{~s}, 3 \mathrm{H}, \mathrm{C}_{7}-\mathrm{CH}_{3}\right), 0.89\left(\mathrm{~s}, 3 \mathrm{H}, \mathrm{C}_{7}-\mathrm{CH}_{3}\right) ;{ }^{13} \mathrm{C}-\mathrm{NMR}$ $\delta=172.19\left(C_{11}\right), 161.23\left(C_{5}\right), 147.61\left(C_{3}\right), 118.43\left(C_{4}\right), 28.01\left(C_{6}\right), 27.14\left(C_{9}\right), 26.69\left(C_{12}\right), 23.70\left(C_{2}\right), 23.04$ $\left(\mathrm{C}_{1}\right), 21.59\left(\mathrm{C}_{10}\right), 20.97\left(\mathrm{C}_{7}\right), 14.52\left(\mathrm{C}_{8}\right), 9.21\left(\mathrm{C}_{13}\right)$; ESI-MS m/z: $222.17[\mathrm{M}+\mathrm{H}]^{+}$. Anal. Calcd. For $\mathrm{C}_{13} \mathrm{H}_{19} \mathrm{NO}_{2}$ (221.30): C, 70.56; H, 8.65; N, 6.33. Found: $\mathrm{C}, 70.59 ; \mathrm{H}, 8.64 ; \mathrm{N}, 6.34$.

(Z)-3-Caren-5-one O-n-butanoyl oxime ((Z)-4v). Yellow liquid. Yield: $65.7 \%$. UV-Vis $(\mathrm{EtOH}) \lambda_{\max }(\log \varepsilon)$ : $243.60(4.19) \mathrm{nm}$; IR (thin film, $\left.\mathrm{cm}^{-1}\right)$ : IR $\left(\mathrm{KBr}, \mathrm{cm}^{-1}\right)$ : 1757 (s, C=O), $1653(\mathrm{~m}, \mathrm{C}=\mathrm{N}) ;{ }^{1} \mathrm{H}-\mathrm{NMR} \delta=6.06$ $\left(\mathrm{d}, J=0.9 \mathrm{~Hz}, 1 \mathrm{H}, \mathrm{C}_{4}-\mathrm{H}\right), 2.51\left(\mathrm{dd}, J=20.3,8.3 \mathrm{~Hz}, 1 \mathrm{H}, \mathrm{C}_{2}-\mathrm{H}_{\mathrm{a}}\right), 2.44\left(\mathrm{t}, J=7.3 \mathrm{~Hz}, 2 \mathrm{H}, \mathrm{C}_{12}-\mathrm{H}\right), 2.17(\mathrm{~d}$, $\left.J=20.3 \mathrm{~Hz}, 1 \mathrm{H}, \mathrm{C}_{2}-\mathrm{H}_{\mathrm{b}}\right), 1.90\left(\mathrm{~d}, J=8.2 \mathrm{~Hz}, 1 \mathrm{H}, \mathrm{C}_{6}-\mathrm{H}\right), 1.81\left(\mathrm{~s}, 3 \mathrm{H}, \mathrm{C}_{3}-\mathrm{CH}_{3}\right), 1.75$ (ddd, $J=14.8,7.4$, $\left.2.7 \mathrm{~Hz}, 2 \mathrm{H}, \mathrm{C}_{13}-\mathrm{H}\right), 1.27\left(\mathrm{t}, J=8.2,1 \mathrm{H}, \mathrm{C}_{1}-\mathrm{H}\right), 1.23\left(\mathrm{~s}, 3 \mathrm{H}, \mathrm{C}_{7}-\mathrm{CH}_{3}\right), 1.01\left(\mathrm{t}, J=7.4 \mathrm{~Hz}, 3 \mathrm{H}, \mathrm{C}_{14}-\mathrm{H}\right), 0.89$ $\left(\mathrm{s}, 3 \mathrm{H}, \mathrm{C}_{7}-\mathrm{CH}_{3}\right) .{ }^{13} \mathrm{C}-\mathrm{NMR} \delta=171.33\left(\mathrm{C}_{11}\right), 161.21\left(\mathrm{C}_{5}\right), 147.62\left(\mathrm{C}_{3}\right), 118.44\left(\mathrm{C}_{4}\right), 35.22\left(\mathrm{C}_{12}\right), 28.01\left(\mathrm{C}_{6}\right)$, $27.13\left(\mathrm{C}_{9}\right), 23.70\left(\mathrm{C}_{2}\right), 23.03\left(\mathrm{C}_{1}\right), 21.58\left(\mathrm{C}_{10}\right), 21.00\left(\mathrm{C}_{7}\right), 18.54\left(\mathrm{C}_{13}\right), 14.52\left(\mathrm{C}_{8}\right), 13.76\left(\mathrm{C}_{14}\right)$; ESI-MS $m / z$ : $236.19[\mathrm{M}+\mathrm{H}]^{+}$. Anal. Calcd. For $\mathrm{C}_{14} \mathrm{H}_{21} \mathrm{NO}_{2}$ (235.32): $\mathrm{C}, 71.46 ; \mathrm{H}, 8.99 ; \mathrm{N}, 5.95$. Found: $\mathrm{C}, 71.44 ; \mathrm{H}$, 8.97; N, 5.93 .

(Z)-3-Caren-5-one O-n-pentanoyl oxime ((Z)-4w). Yellow liquid. Yield: 60.0\%. UV-Vis (EtOH) $\lambda_{\max }$ $(\log \varepsilon): 243.40(4.32) \mathrm{nm}$; IR (thin film, $\left.\mathrm{cm}^{-1}\right): 1769(\mathrm{~s}, \mathrm{C}=\mathrm{O}), 1656(\mathrm{~m}, \mathrm{C}=\mathrm{N}), 763(\mathrm{~m}), 733\left(\mathrm{~m},-\left(\mathrm{CH}_{2}\right)_{n^{-}}\right.$, $n \geq 4) ;{ }^{1} \mathrm{H}-\mathrm{NMR} \delta=6.06\left(\mathrm{~s}, 1 \mathrm{H}, \mathrm{C}_{4}-\mathrm{H}\right), 2.51\left(\mathrm{dd}, J=20.3,7.7 \mathrm{~Hz}, 1 \mathrm{H}, \mathrm{C}_{2}-\mathrm{H}_{\mathrm{a}}\right), 2.46\left(\mathrm{t}, 2 \mathrm{H}, \mathrm{C}_{12}-\mathrm{H}\right), 2.18$ $\left(\mathrm{d}, J=19.6 \mathrm{~Hz}, 1 \mathrm{H}, \mathrm{C}_{2}-\mathrm{H}_{\mathrm{b}}\right), 1.90\left(\mathrm{~d}, J=8.2 \mathrm{~Hz}, 1 \mathrm{H}, \mathrm{C}_{6}-\mathrm{H}\right), 1.81\left(\mathrm{~s}, 3 \mathrm{H}, \mathrm{C}_{3}-\mathrm{CH}_{3}\right), 1.73-1.68(\mathrm{~m}, 2 \mathrm{H}$, $\left.\mathrm{C}_{13}-\mathrm{H}\right), 1.41\left(\mathrm{dt}, J=14.8,7.4 \mathrm{~Hz}, 2 \mathrm{H}, \mathrm{C}_{14}-\mathrm{H}\right), 1.27\left(\mathrm{t}, J=8.5 \mathrm{~Hz}, 1 \mathrm{H}, \mathrm{C}_{1}-\mathrm{H}\right), 1.23\left(\mathrm{~s}, 3 \mathrm{H}, \mathrm{C}_{7}-\mathrm{CH}_{3}\right), 0.94$ $\left(\mathrm{t}, J=7.4 \mathrm{~Hz}, 3 \mathrm{H}, \mathrm{C}_{15}-\mathrm{H}\right), 0.89\left(\mathrm{~s}, 3 \mathrm{H}, \mathrm{C}_{7}-\mathrm{CH}_{3}\right) .{ }^{13} \mathrm{C}-\mathrm{NMR} \delta=171.51\left(\mathrm{C}_{11}\right), 161.20\left(\mathrm{C}_{5}\right), 147.61\left(\mathrm{C}_{3}\right)$, $118.44\left(\mathrm{C}_{4}\right), 33.06\left(\mathrm{C}_{12}\right), 28.02\left(\mathrm{C}_{6}\right), 27.13\left(\mathrm{C}_{9}, \mathrm{C}_{13}\right), 23.70\left(\mathrm{C}_{2}\right), 23.03\left(\mathrm{C}_{1}\right), 22.29\left(\mathrm{C}_{7}\right), 21.59\left(\mathrm{C}_{10}\right), 21.01$ $\left(\mathrm{C}_{14}\right), 14.53\left(\mathrm{C}_{8}\right), 13.72\left(\mathrm{C}_{15}\right)$; ESI-MS $m / z: 250.08$ [M + H] $]^{+}$. Anal. Calcd. For $\mathrm{C}_{15} \mathrm{H}_{23} \mathrm{NO}_{2}$ (249.35): $\mathrm{C}$, 72.25; H, 9.30; N, 5.62. Found: C, 72.23; H, 9.31; N, 5.60.

(E)-3-Caren-5-one O-(4-fluorobenzoyl) oxime ((E)-4f'). Pale yellow liqiud, yield: 60.0\%. UV-Vis (EtOH) $\lambda_{\max }(\log \varepsilon): 256.00(3.97) \mathrm{nm}, 229.70(4.21) \mathrm{nm}$; IR (thin film, $\mathrm{cm}^{-1}$ ): 3078, 3009 (w, Ar-H, =C-H), 1748 $(\mathrm{s}, \mathrm{C}=\mathrm{O}), 1679(\mathrm{~m}, \mathrm{C}=\mathrm{N}) ;{ }^{1} \mathrm{H}-\mathrm{NMR} \delta=8.11\left(\mathrm{dd}, J=8.8,5.5 \mathrm{~Hz}, 2 \mathrm{H}, \mathrm{C}_{13}-\mathrm{H}, \mathrm{C}_{17}-\mathrm{H}\right), 7.15(\mathrm{t}, J=8.6 \mathrm{~Hz}$, $\left.2 \mathrm{H}, \mathrm{C}_{14}-\mathrm{H}, \mathrm{C}_{16}-\mathrm{H}\right), 6.61\left(\mathrm{~s}, 1 \mathrm{H}, \mathrm{C}_{4}-\mathrm{H}\right), 2.58\left(\mathrm{dd}, J=20.7,8.0 \mathrm{~Hz}, 1 \mathrm{H}, \mathrm{C}_{2}-\mathrm{H}_{\mathrm{a}}\right), 2.27(\mathrm{~d}, J=20.7 \mathrm{~Hz}, 1 \mathrm{H}$, $\left.\mathrm{C}_{2}-\mathrm{H}_{\mathrm{b}}\right), 1.91\left(\mathrm{~s}, 3 \mathrm{H}, \mathrm{C}_{3}-\mathrm{CH}_{3}\right), 1.84\left(\mathrm{~d}, J=8.6 \mathrm{~Hz}, 1 \mathrm{H}, \mathrm{C}_{6}-\mathrm{H}\right), 1.33\left(\mathrm{~d}, J=8.0 \mathrm{~Hz}, 1 \mathrm{H}, \mathrm{C}_{1}-\mathrm{H}\right), 1.19(\mathrm{~s}, 3 \mathrm{H}$, $\left.\mathrm{C}_{7}-\mathrm{CH}_{3}\right), 0.94\left(\mathrm{~s}, 3 \mathrm{H}, \mathrm{C}_{7}-\mathrm{CH}_{3}\right) ;{ }^{13} \mathrm{C}-\mathrm{NMR} \delta 166.60\left(\mathrm{C}_{11}\right), 164.92\left(\mathrm{C}_{5}\right), 163.10\left(\mathrm{C}_{15}\right), 159.43\left(\mathrm{C}_{12}\right), 152.56$ $\left(C_{3}\right), 132.06\left(C_{13}\right.$ or $\left.C_{17}\right), 125.84\left(C_{13}\right.$ or $\left.C_{17}\right), 115.72\left(C_{14}\right.$ or $\left.C_{16}\right), 115.58\left(C_{14}\right.$ or $\left.C_{16}\right), 113.16\left(C_{4}\right), 28.12$ $\left(C_{6}\right), 28.01\left(C_{9}\right), 24.23\left(C_{2}\right), 23.77\left(C_{1}\right), 22.35\left(C_{10}\right), 20.57\left(C_{7}\right), 14.13\left(C_{8}\right)$; ESI-MS $m / z: 288.16\left[\mathrm{M}+\mathrm{H}^{+}\right.$. Anal. Calcd. For $\mathrm{C}_{17} \mathrm{H}_{18} \mathrm{FNO}_{2}$ (287.33): C, 71.06; H, 6.31; N, 4.87. Found: $\mathrm{C}, 71.02 ; \mathrm{H}, 6.32 ; \mathrm{N}, 4.85$.

(E)-3-Caren-5-one O-(2, 3-dichlorobenzoyl) oxime ((E)-41'). Yellow liquid. Yield: 60.8\%. UV-Vis (EtOH) $\lambda_{\max }\left(\log \varepsilon\right.$ ):253.00 (4.26) nm; IR (thin film, $\mathrm{cm}^{-1}$ ): 3071, 3009 (w, Ar-H, =C-H), 1759 (s, C=O), 1649 $(\mathrm{m}, \mathrm{C}=\mathrm{N}) ;{ }^{1} \mathrm{H}-\mathrm{NMR} \delta=7.67\left(\mathrm{dd}, J=7.7,1.5 \mathrm{~Hz}, 1 \mathrm{H}, \mathrm{C}_{17}-\mathrm{H}\right), 7.61\left(\mathrm{dd}, J=8.0,1.5 \mathrm{~Hz}, 1 \mathrm{H}, \mathrm{C}_{15}-\mathrm{H}\right), 7.30(\mathrm{t}$, $\left.J=7.9 \mathrm{~Hz}, 1 \mathrm{H}, \mathrm{C}_{16}-\mathrm{H}\right), 6.58\left(\mathrm{~s}, 1 \mathrm{H}, \mathrm{C}_{4}-\mathrm{H}\right), 2.57\left(\mathrm{dd}, J=20.8,8.1 \mathrm{~Hz}, 1 \mathrm{H}, \mathrm{C}_{5}-\mathrm{H}_{\mathrm{a}}\right), 2.26(\mathrm{~d}, J=20.8 \mathrm{~Hz}, 1 \mathrm{H}$, $\left.\mathrm{C}_{5}-\mathrm{H}_{\mathrm{b}}\right), 1.87\left(\mathrm{~s}, 3 \mathrm{H}, \mathrm{C}_{3}-\mathrm{CH}_{3}\right), 1.82\left(\mathrm{~d}, J=8.5 \mathrm{~Hz}, 1 \mathrm{H}, \mathrm{C}_{6}-\mathrm{H}\right), 1.35\left(\mathrm{t}, J=8.3 \mathrm{~Hz}, 1 \mathrm{H}, \mathrm{C}_{1}-\mathrm{H}\right), 1.19(\mathrm{~s}, 3 \mathrm{H}$, $\left.\mathrm{C}_{7}-\mathrm{CH}_{3}\right), 0.93\left(\mathrm{~s}, 3 \mathrm{H}, \mathrm{C}_{7}-\mathrm{CH}_{3}\right),{ }^{13} \mathrm{C}-\mathrm{NMR} \delta=163.37\left(\mathrm{C}_{11}\right), 159.97\left(\mathrm{C}_{2}\right), 153.10\left(\mathrm{C}_{3}\right), 134.40\left(\mathrm{C}_{12}\right), 133.00$ $\left(\mathrm{C}_{15}\right), 132.86\left(\mathrm{C}_{14}\right), 131.27\left(\mathrm{C}_{13}\right), 129.12\left(\mathrm{C}_{17}\right), 127.32\left(\mathrm{C}_{16}\right), 113.42\left(\mathrm{C}_{4}\right), 28.11\left(\mathrm{C}_{1}\right), 28.01\left(\mathrm{C}_{9}\right), 24.17$ $\left(\mathrm{C}_{5}\right), 23.67\left(\mathrm{C}_{6}\right), 22.47\left(\mathrm{C}_{10}\right), 20.68\left(\mathrm{C}_{7}\right), 14.11\left(\mathrm{C}_{8}\right)$; ESI-MS $m / z: 337.83[\mathrm{M}-\mathrm{H}]^{-} .$. For $\mathrm{C}_{17} \mathrm{H}_{17} \mathrm{Cl}_{2} \mathrm{NO}_{2}$ (338.23): C, 60.37; H, 5.07; N, 4.14. Found: C, 60.39; H, 5.06; N, 4.13.

(E)-3-Caren-5-one O- $\beta$-pyridylcarbonyl oxime $\left((E)-4 \mathbf{r}^{\prime}\right)$. White solid. Yield 78.3\%, m.p.: $108.5-109.3^{\circ} \mathrm{C}$. UV-Vis (EtOH) $\lambda_{\max }(\log \varepsilon): 262.50(4.07) \mathrm{nm}, 223.40$ (4.12) nm; IR $\left(\mathrm{KBr}, \mathrm{cm}^{-1}\right): 3092,3057$ (w, Ar-H, 
$=\mathrm{C}-\mathrm{H}), 1750(\mathrm{~s}, \mathrm{C}=\mathrm{O}), 1635(\mathrm{~m}, \mathrm{C}=\mathrm{N}) ;{ }^{1} \mathrm{H}-\mathrm{NMR} \delta=9.29\left(\mathrm{~s}, 1 \mathrm{H}, \mathrm{C}_{13}-\mathrm{H}\right), 8.80\left(\mathrm{~d}, J=4.8 \mathrm{~Hz}, 1 \mathrm{H}, \mathrm{C}_{14}-\mathrm{H}\right)$, $8.38\left(\mathrm{~d}, J=7.9 \mathrm{~Hz}, 1 \mathrm{H}, \mathrm{C}_{16}-\mathrm{H}\right), 7.45\left(\mathrm{dd}, J=8.3,5.2 \mathrm{~Hz}, 1 \mathrm{H}, \mathrm{C}_{15}-\mathrm{H}\right), 6.63\left(\mathrm{~s}, 1 \mathrm{H}, \mathrm{C}_{4}-\mathrm{H}\right), 2.60(\mathrm{dd}, J=20.8$, $\left.8.1 \mathrm{~Hz}, 1 \mathrm{H}, \mathrm{C}_{2}-\mathrm{H}_{\mathrm{a}}\right), 2.29\left(\mathrm{~d}, J=20.8 \mathrm{~Hz}, 1 \mathrm{H}, \mathrm{C}_{2}-\mathrm{H}_{\mathrm{b}}\right), 1.92\left(\mathrm{~s}, 3 \mathrm{H}, \mathrm{C}_{3}-\mathrm{CH}_{3}\right), 1.84(\mathrm{~d}, J=8.5 \mathrm{~Hz}, 1 \mathrm{H}$, $\left.\mathrm{C}_{3}-\mathrm{CH}_{3}\right), 1.36\left(\mathrm{t}, J=8.2 \mathrm{~Hz}, 1 \mathrm{H}, \mathrm{C}_{1}-\mathrm{H}\right), 1.20\left(\mathrm{~s}, 3 \mathrm{H}, \mathrm{C}_{7}-\mathrm{CH}_{3}\right), 0.95\left(\mathrm{~s}, 3 \mathrm{H}, \mathrm{C}_{7}-\mathrm{CH}_{3}\right) ;{ }^{13} \mathrm{C}-\mathrm{NMR} \delta=162.71$ $\left(\mathrm{C}_{11}\right), 159.90\left(\mathrm{C}_{5}\right), 153.42\left(\mathrm{C}_{3}\right), 153.19\left(\mathrm{C}_{14}\right), 150.55\left(\mathrm{C}_{13}\right), 137.24\left(\mathrm{C}_{16}\right), 125.77\left(\mathrm{C}_{12}\right), 123.51\left(\mathrm{C}_{15}\right), 113.03$ $\left(\mathrm{C}_{4}\right), 28.15\left(\mathrm{C}_{6}\right), 28.01\left(\mathrm{C}_{9}\right), 24.24\left(\mathrm{C}_{2}\right), 23.74\left(\mathrm{C}_{6}\right), 22.43\left(\mathrm{C}_{10}\right), 20.68\left(\mathrm{C}_{7}\right), 14.13\left(\mathrm{C}_{8}\right)$; ESI-MS m/z: 270.96 $[\mathrm{M}+\mathrm{H}]^{+}$. Anal. Calcd. For $\mathrm{C}_{16} \mathrm{H}_{18} \mathrm{~N}_{2} \mathrm{O}_{2}$ (270.33): C, 71.09; H, 6.71; N, 10.36. Found: C, 71.11; H, 6.69; $\mathrm{N}, 10.37$.

\subsection{Antifungal Activity Test}

Antifungal activity of the target compounds was perform in vitro method [40]. The tested compound was dissolved in acetone. Sorporl-144 $(200 \mu \mathrm{g} / \mathrm{mL})$ emulsifier was added to dilute the solution to $500 \mu \mathrm{g} / \mathrm{mL}$. Then, $1 \mathrm{~mL}$ solution of the tested compound was poured into a culture plate, and then $9 \mathrm{~mL}$ PSA culture medium was added to obtain the flat containing $50 \mu \mathrm{g} / \mathrm{mL}$ tested compound. A bacterium tray of 5-mm diameter cut along the external edge of the mycelium was transferred to the flat containing the tested compound and put in equilateral triangular style in duplicate. Later, the culture plate was cultured at $24 \pm 1{ }^{\circ} \mathrm{C}$ and the expanded diameter of the bacterium tray was measured after $48 \mathrm{~h}$ and compared with that treated with aseptic distilled water to calculate the relative inhibition percentage:

$$
\text { Relative inhibitory rate }(\%)=(C K-P T) / C K \times 100 \%
$$

where $C K$ is the extended diameter of the circle of mycelium during the blank assay and $P T$ is the extended diameter of the circle of mycelium during testing.

\section{Conclusions}

Twenty-four novel 3-caren-5-one oxime esters were designed, synthesized, characterized, and evaluated for their antifungal activity. As a result, at $50 \mu \mathrm{g} / \mathrm{mL}$, the target compounds exhibited best antifungal activity against $P$. piricola, in which compounds $(Z)-\mathbf{4 r},(Z)-\mathbf{4 q},(E)-\mathbf{4} \mathbf{f}^{\prime},(Z)-\mathbf{4 i},(Z)-\mathbf{4} \mathbf{j}$, and (Z)-4p had inhibition rates of $97.1 \%, 87.4 \%, 87.4 \%, 85.0 \%, 81.9 \%$ and $77.7 \%$, respectively, showing better antifungal activity than that of the commercial fungicide chlorothanil. Also, compound (Z)-4r displayed remarkable antifungal activity against all the tested fungi, with inhibition rates of $76.7 \%$, $82.7 \%, 97.1 \%, 66.3 \%, 74.7 \%, 93.9 \%, 76.7 \%$ and $93.3 \%$, respectively, showing better or comparable antifungal activity than that of the commercial fungicide chlorothanil. Thus, compound $(Z)-4 \mathbf{r}$ is a lead compound worthy of further investigation. Besides, $E-Z$ isomers of the target oxime esters were found to show obvious difference in antifungal activity. It is very meaningful and require further studies in photoisomerization and drug resistance.

Supplementary Materials: Supplementary materials are available online.

Acknowledgments: This work was supported by the National Natural Science Foundation of China (No. 31560194). The authors are grateful to the State Key Laboratory of Element-organic Chemistry, Nankai University, China, for the bioassay test.

Author Contributions: Min Huang carried out the experimental work, participated in the discussion of biological activities, and wrote the paper; Wen-Gui Duan and Gui-Shan Lin constructed the target compound structure, designed the experimental scheme, directed and supervised the whole experimentation, discussed the biological activities, and revised the paper; Kun Li explored the preparation of 3-carene-5-one; Qiong Hu participated in the discussion of biological activities.

Conflicts of Interest: The authors declare no conflict of interest. 


\section{References}

1. Qiu, C.L.; Smuts, J.; Schug, K.A. Analysis of terpenes and turpentines using gas chromatography with vacuum ultraviolet detection. J. Sep. Sci. 2017, 40, 869-877. [CrossRef] [PubMed]

2. Garcia, G.; Tissandie, L.; Filippi, J.J.; Tomi, F. New pinane derivatives found in essential oils of Calocedrus decurrens. Molecules 2017, 22, 921. [CrossRef] [PubMed]

3. Gupta, M.; Rout, P.K.; Misra, L.N.; Gupta, P.; Singh, N.; Darokar, M.P.; Saikia, D.; Singh, S.C.; Bhakuni, R.S. Chemical composition and bioactivity of Boswellia serrata Roxb. essential oil in relation to geographical variation. Plant Biosyst. 2017, 151, 623-629. [CrossRef]

4. Ibrahim, T.A.; El-Hela, A.A.; El-Hefnawy, H.M.; Al-Taweel, A.M.; Perveen, S. Chemical composition and antimicrobial activities of essential oils of some coniferous plants cultivated in Egypt. Iran. J. Pharm. Res. 2017, 16, 328-337. [PubMed]

5. Li, Z.; Wang, M.; Peng, L. Chemical composition analysis of essential oil from Mosla chinensis maxim. cv. Jiangxiangru and inhibitory activity of the oil and its major constituents on biofilm formation of Staphylococcus aureus. Food Sci. 2016, 37, 138-143.

6. Smeriglio, A.; Denaro, M.; Barreca, D.; Calderaro, A.; Bisignano, C.; Ginestra, G.; Bellocco, E.; Trombetta, D. In vitro evaluation of the antioxidant, cytoprotective, and antimicrobial properties of essential oil from Pistacia vera L. Variety Bronte Hull. Int. J. Mol. Sci. 2017, 18, 1212. [CrossRef] [PubMed]

7. Pan, C.; Lin, J.; Han, B. Evaluation of attraction of volatile semiochemicals from tea shoots to the mymarid Stethynium empoascae. Acta Ecol. Sin. 2016, 36, 3785-3795.

8. Gray, C.A.; Runyon, J.B.; Jenkins, M.J.; Giunta, A.D. Mountain pine beetles use volatile cues to locate host limber pine and avoid non-host great basin bristlecone pine. PLoS ONE 2015, 10, 1371-1384. [CrossRef] [PubMed]

9. Wajs-Bonikowska, A.; Stobiecka, A.; Bonikowski, R.; Krajewska, A.; Sikora, M.; Kula, J. A comparative study on composition and antioxidant activities of supercritical carbon dioxide, hexane and ethanol extracts from blackberry (Rubus fruticosus) growing in Poland. J. Sci. Food Agric. 2017, 97, 3576-3583. [CrossRef] [PubMed]

10. Hu, W.; Zhang, N.; Chen, H.; Zhong, B.; Yang, A.; Kuang, F.; Ouyang, Z.; Chun, J. Fumigant activity of sweet orange essential oil fractions against red imported fire ants (Hymenoptera: Formicidae). J. Econ. Entomol. 2017, 4, 1556-1562. [CrossRef] [PubMed]

11. Bolli, M.H.; Muller, C.; Mathys, B.; Abele, S.; Birker, M.; Bravo, R.; Bur, D.; Hess, P.; Kohl, C.; Lehmann, D.; et al. Novel S1P 1 receptor agonists-Part 1: From pyrazoles to thiophenes. J. Med. Chem. 2013, 56, 9737-9755. [CrossRef] [PubMed]

12. Jin, Y.; Yeh, C.H.; Kuttruff, C.A.; Jorgensen, L.; Dunstl, G.; Felding, J.; Natarajan, S.R.; Baran, P.S. C-H oxidation of ingenanes enables potent and selective protein kinase $\mathrm{C}$ isoform activation. Angew. Chem. Int. Ed. 2015, 54, 14044-14048. [CrossRef] [PubMed]

13. Kawamura, S.; Chu, H.; Felding, J.; Baran, P.S. Nineteen-step total synthesis of (+)-phorbol. Nature 2016, 532, 90-93. [CrossRef] [PubMed]

14. Liang, X.; Grue-Sorensen, G.; Mansson, K.; Vedso, P.; Soor, A.; Stahlhut, M.; Bertelsen, M.; Engell, K.M.; Hogberg, T. Syntheses, biological evaluation and SAR of ingenol mebutate analogues for treatment of actinic keratosis and non-melanoma skin cancer. Bioorg. Med. Chem. Lett. 2013, 23, 5624-5629. [CrossRef] [PubMed]

15. McKerrall, S.J.; Joergensen, L.; Kuttruff, C.A.; Ungeheuer, F.; Baran, P.S. Development of a concise synthesis of (-)-ingenol. J. Am. Chem. Soc. 2014, 136, 5799-5810. [CrossRef] [PubMed]

16. Dai, H.; Li, H.; Jin, Z.C.; Liu, W.Y.; Xiao, Y.; He, H.; Wang, Q.M.; Shi, Y.J. Synthesis and bioactivity of novel 1-methyl-3-trifluoromethyl-5-subsituent-1H-pyrazole-4-carbaldehyde-O-(4-trifluoromethylbenzoyl)oximes. Chin. J. Org. Chem. 2016, 36, 185-190. [CrossRef]

17. Li, Q.; Huang, X.B.; Li, S.C.; Ma, J.C.; Lv, M.; Xu, H. Semisynthesis of ssters of fraxinellone C4/10-oxime and their pesticidal activities. J. Agric. Food Chem. 2016, 64, 5472-5478. [CrossRef] [PubMed]

18. Li, T.G.; Liu, J.P.; Han, J.T.; Fu, B.; Wang, D.Q.; Wang, M.G. Synthesis and herbicidal activity of alpha-phenylsulfonylcyclododecanone oxime esters. Chin. J. Org. Chem. 2009, 29, 898-903.

19. Wang, X.; Xia, L.; Xie, Y.; Wang, X.; Xiao, W.; Zhong, X.; Huang, M.; Xue, W. Synthesis and antiviral activities of curcumin derivatives bearing oxime esters moiety. Agrochemicals 2016, 55, 641-646. 
20. Chen, H.P.; Zhao, Z.Z.; Li, Z.H.; Dong, Z.J.; Wei, K.; Bai, X.; Zhang, L.; Wen, C.N.; Feng, T.; Liu, J.K. Novel natural oximes and oxime esters with a vibralactone backbone from the Basidiomycete Boreostereum vibrans. ChemistryOpen 2016, 5, 142-149. [CrossRef] [PubMed]

21. Chen, S.R.; Shen, F.J.; Feng, G.L.; Yuan, R.X. Synthesis and anticancer activity of 4-azasteroidal-20-oxime derivatives. J. Chem. Res. 2015, 39, 527-530. [CrossRef]

22. Hamid, A.A.; Kaushal, T.; Ashraf, R.; Singh, A.; Gupta, A.C.; Prakash, O.; Sarkar, J.; Chanda, D.; Bawankule, D.U.; Khan, F.; et al. (22 $\beta, 25 R)-3 \beta$-Hydroxy-spirost-5-en-7-iminoxy-heptanoic acid exhibits anti-prostate cancer activity through caspase pathway. Steroids 2017, 119, 43-52. [CrossRef] [PubMed]

23. Kucuk, H.B.; Yusufoglu, A.S.; Acik, L.; Aydin, B.; Arslan, L. Synthesis, (E)/(Z)-isomerization, and DNA binding, antibacterial, and antifungal activities of novel oximes and O-substituted oxime ethers. Turk. J. Chem. 2016, 40, 816-829. [CrossRef]

24. Krishnan, K.G.; Sivakumar, R.; Thanikachalam, V. Synthesis, structural characterization and antimicrobial evaluation of some novel piperidin-4-one oxime esters. J. Serb. Chem. Soc. 2015, 80, 1101-1111. [CrossRef]

25. Sammaiah, A.; Kaki, S.S.; Manoj, G.; Poornachandra, Y.; Kumar, C.G.; Prasad, R.B.N. Novel fatty acid esters of apocynin oxime exhibit antimicrobial and antioxidant activities. Eur. J. Lipid Sci. Technol. 2015, 117, 692-700. [CrossRef]

26. Ahluwalia, V.; Kumar, J.; Rana, V.S.; Singh, R.; Sati, O.P.; Walia, S.; Garg, N. Synthesis and antimicrobial activity of esters of 3-ethoxy-4-hydroxybenzaldehyde oxime. Toxicol. Environ. Chem. 2017, 99, 1-9. [CrossRef]

27. Lin, G.S.; Duan, W.G.; Yang, L.X.; Huang, M.; Lei, F.H. Synthesis and antifungal activity of novel myrtenal-based 4-methyl-1,2,4-triazole-thioethers. Molecules 2017, 22, 193. [CrossRef] [PubMed]

28. Li, F.Y.; Wang, X.; Duan, W.G.; Lin, G.S. Synthesis and in vitro anticancer activity of novel dehydroabietic acid-based acylhydrazones. Molecules 2017, 22, 1087. [CrossRef] [PubMed]

29. Chen, N.; Duan, W.; Lin, G.; Liu, L.; Zhang, R.; Li, D. Synthesis and antifungal activity of dehydroabietic acid-based 1,3,4-thiadiazole-thiazolidinone compounds. Mol. Divers. 2016, 20, 897-905. [CrossRef] [PubMed]

30. Ma, X.L.; Li, F.Y.; Duan, W.G.; Liao, J.N.; Lin, Z.D.; Lin, G.S.; Cen, B.; Lei, F.H. Synthesis and antifungal activity of camphoric acid-based acylhydrazone compounds. Holzforschung 2014, 68, 889-895. [CrossRef]

31. Li, F.Y.; Mo, Q.J.; Duan, W.G.; Lin, G.S.; Cen, B.; Chen, N.Y.; Yang, Z.Q. Synthesis and insecticidal activities of N-(5-dehydroabietyl-1,3,4-thiadiazol-2-yl)-benzenesulfonamides. Med. Chem. Res. 2014, 23, 4420-4426. [CrossRef]

32. Lin, G.S.; Ma, C.H.; Duan, W.G.; Cen, B.; Lei, F.H.; Yang, Z.Q. Synthesis and biological activities of $\alpha$-pinene-based dithiadiazoles. Holzforschung 2014, 68, 75-83. [CrossRef]

33. Huang, D.Y.; Duan, W.G.; Lin, G.S.; Bai, X.; Xiao, H.; Yang, Z.Q. Synthesis and antifungal activities of 2-sustituted acylamino-5-( $\alpha$-campholenicaldehyde)-based-1,3,4-thiadiazole compounds. Chem. Ind. For. Prod. 2016, 36, 61-69.

34. Alfayate, A.; Márquez-Álvarez, C.; Grande-Casas, M.; Bernardo-Maestro, B.; Sánchez-Sánchez, M.; Pérez-Pariente, J. Enhanced catalytic activity of TAPO-5 in the oxidation of cyclohexene with hydrogen peroxide under anhydrous conditions. Catal. Today 2013, 213, 211-218. [CrossRef]

35. Vanelderen, P.; Snyder, B.E.; Tsai, M.L.; Hadt, R.G.; Vancauwenbergh, J.; Coussens, O.; Schoonheydt, R.A.; Sels, B.F.; Solomon, E.I. Spectroscopic definition of the copper active sites in mordenite: Selective methane oxidation. J. Am. Chem. Soc. 2015, 137, 6383-6392. [CrossRef] [PubMed]

36. Narsimhan, K.; Iyoki, K.; Dinh, K.; Roman-Leshkov, Y. Catalytic oxidation of methane into methanol over copper-exchanged zeolites with oxygen at low temperature. ACS Cent. Sci. 2016, 2, 424-429. [CrossRef] [PubMed]

37. Chanquía, C.M.; Cánepa, A.L.; Bazán-Aguirre, J.; Sapag, K.; Rodríguez-Castellón, E.; Reyes, P.; Herrero, E.R.; Casuscelli, S.G.; Eimer, G.A. Copper-containing spherical mesoporous silicates prepared by template-ion exchange: A multitechnique characterization and oxidation properties. Microporous Mesoporous Mater. 2012, 151, 2-12. [CrossRef]

38. Krishnan, K.G.; Sivakumar, R.; Thanikachalam, V. Synthesis and spectral study of some novel oxime esters derived from 3-azabicyclo 3.3.1 nonan-9-one oxime. Lett. Org. Chem. 2015, 12, 31-37. [CrossRef]

39. Fan, M.G.; Fang, J.L.; Zhou, L.C.; Li, W.L.; Li, B.; Xing, J.M.; Liu, Z.L. Distribution of the Cu ions in the CuHY zeolite and its performance of desulfurization. Chem. J. Chin. Univ. 2008, 29, 1834-1840. 
40. Su, N.N.; Li, Y.; Yu, S.J.; Zhang, X.; Liu, X.H.; Zhao, W.G. Microwave-assisted synthesis of some novel 1,2,3-triazoles by click chemistry, and their biological activity. Res. Chem. Intermed. 2012, 39, 759-766. [CrossRef]

Sample Availability: Samples of the compounds 2, (Z)-3a, (E)-3b, (Z)-4a-4w, and $(E)-\mathbf{4} \mathbf{f}^{\prime}, \mathbf{4} \mathbf{1}^{\prime}, \mathbf{4} \mathbf{r}^{\prime}$ are available from the authors.

(C) 2017 by the authors. Licensee MDPI, Basel, Switzerland. This article is an open access article distributed under the terms and conditions of the Creative Commons Attribution (CC BY) license (http://creativecommons.org/licenses/by/4.0/). 